\title{
Crystalline Color Superconductivity
}

\author{
Mark Alford, Jeffrey A. Bowers, and Krishna Rajagopal \\ Center for Theoretical Physics \\ Massachusetts Institute of Technology \\ Cambridge, MA 02139
}

August 18, 2000

MIT-CTP-3012

\begin{abstract}
In any context in which color superconductivity arises in nature, it is likely to involve pairing between species of quarks with differing chemical potentials. For suitable values of the differences between chemical potentials, Cooper pairs with nonzero total momentum are favored, as was first realized by Larkin, Ovchinnikov, Fulde and Ferrell (LOFF). Condensates of this sort spontaneously break translational and rotational invariance, leading to gaps which vary periodically in a crystalline pattern. Unlike the original LOFF state, these crystalline quark matter condensates include both spin zero and spin one Cooper pairs. We explore the range of parameters for which crystalline color superconductivity arises in the QCD phase diagram. If in some shell within the quark matter core of a neutron star (or within a strange quark star) the quark number densities are such that crystalline color superconductivity arises, rotational vortices may be pinned in this shell, making it a locus for glitch phenomena.
\end{abstract}




\section{Overview}

The attraction between two quarks which are anti-symmetric in color renders cold dense quark matter unstable to the formation of quark Cooper pairs in a color superconducting state [1, 2, 3, 4, 5, 6, 7]. If two (or more) different quark flavors are involved, and their Fermi momenta are the same, they pair as in the standard BCS state. The pairing is guaranteed because in the absence of an interaction each pair costs no free energy - each quark can be created at its Fermi surface - and the interaction then makes the system unstable against formation of a condensate of pairs.

In this paper we study the situation, generic in the real world, where the Fermi momenta of the two species are different. If the Fermi momenta are far apart, no pairing between the species is possible. The transition between the BCS and unpaired states as the splitting between Fermi momenta increases has been studied in electron [8] and QCD [9, 10, 11] superconductors, assuming that no other state intervenes. However, there is good reason to think that another state can occur. This is the "LOFF" state, first explored by Larkin and Ovchinnikov [12 and Fulde and Ferrell [13] in the context of electron superconductivity in the presence of magnetic impurities. They found that near the unpairing transition, it is favorable to form a crystalline state in which the Cooper pairs have nonzero momentum. This is favored because it gives rise to a region of phase space where each of the two quarks in a pair can be close to its Fermi surface, and such pairs can be created at low cost in free energy.

We study the pairing between two species whose chemical potentials differ by $2 \delta \mu$ and find that for a large class of interactions there is a window of $\delta \mu$ within which states of the LOFF type are preferred over the BCS and unpaired states. This has important ramifications for compact star phenomenology, since it means that there may be a layer of crystalline quark matter inside the star. This could pin rotational vortices, and lead to the kind of glitch phenomena that have up to now been thought of as uniquely associated with the nuclear crust of neutron stars.

In Section 2, we give a more detailed introduction to the BCS and LOFF color superconducting states, and their possible astrophysical applications. In Section 3, we describe the LOFF state in quark matter with $\delta \mu \neq 0$. We note in particular that, unlike in the original LOFF context, there is pairing both in $J=0$ and $J=1$ channels. In Section 1 , we derive the gap equation for the LOFF state for a model Hamiltonian in which the full QCD interaction is replaced by a four-fermion interaction with the quantum numbers of single gluon exchange. In Section 5, we use the gap equation to evaluate the range of $\delta \mu$ within which the LOFF state arises. We will see that at low $\delta \mu$ the translationally invariant BCS state, with gap $\Delta_{0}$, is favored. At $\delta \mu_{1}$ there is a first order transition to the LOFF paired state, which breaks translational symmetry. At $\delta \mu_{2}$ all pairing disappears, and translational symmetry is restored at a phase transition which is second order in mean field theory. In the weak-coupling limit, in which $\Delta_{0} \ll \mu$, we find values 
of $\delta \mu_{1}$ and $\delta \mu_{2}$ which are in quantitative agreement with those obtained by LOFF. This agreement occurs only because we have chosen an interaction which is neither attractive nor repulsive in the $J=1$ channel, making the $J=1$ component of our LOFF condensate irrelevant in the gap equation. In Section 6, we consider a more general Hamiltonian in which the couplings corresponding to electric and magnetic gluon exchange can be separately tuned. This leads to interactions in both $J=0$ and $J=1$ channels, and we show how it affects the range of $\delta \mu$ within which the LOFF state arises. In Section 7, we outline future work which follows immediately from what we have done and look farther ahead toward possible astrophysical consequences of crystalline color superconductivity.

We recommend that the astrophysically inclined reader, interested primarily in the consequences of our results, read Sections 2 and 7 , skipping those in between.

\section{Introduction}

\subsection{Astrophysical applications of color superconductivity}

Our current understanding of the color superconducting state of quark matter leads us to believe that it may occur naturally in compact stars. The critical temperature below which quark matter is a color superconductor is generally estimated to be of order $10-50 \mathrm{MeV}$, which suggests that any quark matter which occurs within neutron stars that are more than a few seconds old is in a color superconducting state.

This estimate of the critical temperature comes both from models whose parameters are tuned to reproduce zero density physics [3, 4, 5, 6, 14, 15, 16, 17] and also from weak coupling methods which are quantitatively valid at asymptotically high densities [18, 19, 20, 21, 22, 23, 24, 25, 26, 27, 28, 29], with chemical potentials $\mu \gg 10^{8} \mathrm{MeV}$ [29]. Neither class of methods can be trusted quantitatively for quark number chemical potentials $\mu \sim 400 \mathrm{MeV}$, as appropriate for the quark matter which may occur in the cores of neutron stars. Still, both methods agree that the gaps at the Fermi surface are of order tens to $100 \mathrm{MeV}$, with critical temperatures about half as large.

It is therefore important to look for astrophysical consequences of color superconductivity. As a Fermi surface phenomenon, it has little effect on the equation of state, and hence little effect on the radius of a compact star. There are nevertheless several effects of color superconductivity under active investigation. The color superconductivity of quark matter in neutron stars influences the evolution of magnetic fields within the quark matter [30] (see also [31]). Cooling by neutrino emission is also affected [32] (see also [33]). In quark stars, the physics of the instability to r-mode oscillations is dramatically affected by color superconductivity [34], although this is not the case for neutron stars with quark matter present only in their cores 35, 34. Furthermore, the phase transition at which color superconductivity sets in as a hot proto-neutron star cools may yield 
a detectable signature in the neutrinos received from a supernova [36]. Finally, one goal of the present paper is to motivate an investigation of the possibility that (some) pulsar glitches may originate in quark matter.

If two species of fermion experience an attractive interaction, and their Fermi momenta are the same, they pair in the standard BCS state. The pairing is guaranteed because in the absence of an interaction each pair costs no free energy (each quark can be created at its Fermi surface), and the interaction then makes the system unstable against formation of a condensate of pairs. In the QCD context, if there are two flavors of quarks with equal Fermi momenta, quarks of two colors and two flavors pair [3, 4] while if there are three flavors of quarks, all nine quarks pair in a pattern which locks color and flavor symmetries, breaking chiral symmetry [6, 37]. These idealizations are very instructive, but in any physical context, the up, down and strange quarks will all have different Fermi momenta. To give the reader some sense for typical scales in the problem, we give an illustrative example [30]. The numbers in this paragraph assume that the quarks are noninteracting fermionsclearly a bad assumption - and so should certainly not be construed as precise. Consider quark matter with average quark chemical potential $\mu=400 \mathrm{MeV}$, made of massless up and down quarks and strange quarks with mass $M_{s}=300 \mathrm{MeV}$. $\left(M_{s}\right.$ is a density dependent effective mass; this adds to the uncertainty in its value.) If the strange quark were massless, quark matter consisting of equal parts $u, d$ and $s$ would be electrically neutral. In our illustrative example, on the other hand, electric neutrality requires a nonzero density of electrons, with chemical potential $\mu_{e}=53 \mathrm{MeV}$. Charge neutrality combined with the requirement that the weak interactions are in equilibrium determine all the chemical potentials and Fermi momenta:

$$
\begin{aligned}
\mu_{u}=\mu-\frac{2}{3} \mu_{e} & =365 \mathrm{MeV}, & & p_{F}^{u}=\mu_{u}, \\
\mu_{d}=\mu+\frac{1}{3} \mu_{e} & =418 \mathrm{MeV}, & p_{F}^{d} & =\mu_{d}, \\
\mu_{s}=\mu+\frac{1}{3} \mu_{e} & =418 \mathrm{MeV}, & p_{F}^{s} & =\sqrt{\mu_{s}^{2}-M_{s}^{2}}=290 \mathrm{MeV}, \\
\mu_{e} & =53 \mathrm{MeV}, & p_{F}^{e} & =\mu_{e} .
\end{aligned}
$$

The baryon number density $\rho_{B}=\left(1 / 3 \pi^{2}\right)\left[\left(p_{F}^{u}\right)^{3}+\left(p_{F}^{d}\right)^{3}+\left(p_{F}^{s}\right)^{3}\right]$ is 4 times nuclear matter density.円 As one goes deeper into a neutron star, $\mu$ increases, $M_{s}$ decreases somewhat, and $\mu_{e}$ and all differences between the quark Fermi momenta decrease.I In this paper, we investigate the consequences of pairing between quarks with

\footnotetext{
${ }^{1} \mathrm{Had}$ we chosen $M_{s}=200 \mathrm{MeV}$, we would have obtained $\mu_{e}=24 \mathrm{MeV}, p_{F}^{u}=384 \mathrm{MeV}$, $p_{F}^{d}=408 \mathrm{MeV}, p_{F}^{s}=356 \mathrm{MeV}$ and a $\rho_{B}$ of 5 times nuclear matter density.

${ }^{2}$ Note that in a neutron star with a quark matter core, regions of purely hadronic and purely quark matter are separated by a mixed phase in which neither the hadronic regions nor the quark matter regions are separately charge neutral [38]. The electrically charged quark matter in these regions will have Fermi momenta which differ qualitatively from those in our example, with $p_{F}^{u}$ less than either $p_{F}^{d}$ or $p_{F}^{s}$ [38.
} 
differing Fermi momenta. For simplicity, we restrict our explicit calculations to the case of two massless quarks with differing chemical potentials $\mu_{u}$ and $\mu_{d}$, which we write as

$$
\mu_{d}=\bar{\mu}+\delta \mu ; \quad \mu_{u}=\bar{\mu}-\delta \mu .
$$

We expect similar phenomena to those we describe to arise wherever any one of $\left|p_{F}^{u}-p_{F}^{d}\right|$ or $\left|p_{F}^{u}-p_{F}^{s}\right|$ or $\left|p_{F}^{d}-p_{F}^{s}\right|$ falls within a suitable range, but we leave the investigation of quark matter with $u, d$, and massive $s$ quarks to future work. We also work at zero temperature throughout.

\subsection{Isotropic (non-LOFF) pairing}

In the color superconducting phase for two massless quark flavors at the same chemical potential $\mu$, the condensate consists of quark-quark pairs which are flavor singlets and color $\overline{\mathbf{3}}$ antitriplets (and hence also spin singlets, to obey Pauli statistics). Pairing is of the BCS type: a red up quark of momentum p pairs with a green down quark of momentum $-\mathbf{p}$ of the same helicity, so that the spins are antiparallel. The blue quarks are left unpaired. Such pairing is strongest in the vicinity of the Fermi surface, for || $\mathbf{p}|-\mu| \lesssim \Delta_{0}$, where $\Delta_{0}$ is the BCS gap parameter.

If, instead, the Fermi momenta are sufficiently different, no BCS pairing is possible. It is no longer possible to guarantee that the formation of pairs lowers the free energy, because in the BCS state the two fermions in a pair have equal and opposite momentum, so at most one member of each pair can be created at its Fermi surface. The other member costs non-zero free energy, which the attractive interaction may be unable to compensate.

Assuming that no other state intervenes between the BCS state and the state with no condensate, we can apply the results first derived by Clogston and Chandrasekhar in the context of pairing between spin-up and spin-down electrons with differing Fermi momenta [8]. For small enough $\delta \mu$, the favored BCS state has coincident Fermi surfaces, $p_{F}^{u}=p_{F}^{d}=\bar{\mu}$ because this maximizes the pairing and thus the gain in interaction energy $]^{3}$ We denote the gap in this BCS state by $\Delta_{0}$. The free energy of this BCS state must be compared to that of the unpaired or "normal" state in which the quarks simply distribute themselves in Fermi seas with $p_{F}^{u}=\mu_{u}$, $p_{F}^{d}=\mu_{d}$ and no condensate forms. The BCS state is the stable ground state of the system only when its negative interaction energy offsets the large positive free energy cost associated with forcing the Fermi seas to deviate from their normal state distributions. If $\Delta_{0} \ll \bar{\mu}$ and $\delta \mu \ll \bar{\mu}$, the free energy of the BCS state relative to that of the normal state at a given $\delta \mu$ is

$$
F_{\mathrm{BCS}}-F_{\text {normal }}=\frac{\bar{\mu}^{2}}{3 \pi^{2}}\left(2 \delta \mu^{2}-\Delta_{0}^{2}\right)
$$

\footnotetext{
${ }^{3}$ If one tries to construct a "BCS-like" state which has $p_{F}^{u}=\mu_{u}$ and $p_{F}^{d}=\mu_{d}$ and consequently no pairing for $p_{F}^{u}<p<p_{F}^{d}$, one finds [39] that this state has a higher free energy than the BCS state (in which $p_{F}^{u}=p_{F}^{d}=\bar{\mu}$ ). The gain in free energy associated with choosing $p_{F}^{u}=\mu_{u}$ and $p_{F}^{d}=\mu_{d}$ does not compensate for the lost pairing energy.
} 
The coefficient $1 / 3 \pi^{2}$ depends on the number of fermion species which pair and is appropriate to the case of interest to us. Clogston and Chandrasekhar concluded that the BCS state is favored for $\delta \mu<\delta \mu_{1}=\Delta_{0} / \sqrt{2}$. (The relation $\delta \mu_{1}=\Delta_{0} / \sqrt{2}$ is exact only in the weak-coupling limit in which $\Delta_{0} \ll \bar{\mu}$.) At $\delta \mu=\delta \mu_{1}$, there is a first order phase transition at which the gap parameter drops discontinuously from $\Delta_{0}$ to zero: for $\delta \mu<\delta \mu_{1}$, the system is in the BCS phase, unperturbed from the the $\delta \mu=0$ state.

This analysis is modified in an interesting way at nonzero temperature, as was discussed by Lombardo and Sedrakian in the context of pairing between neutrons and protons in nuclei [40]. Thermal excitations smear out the normal state Fermi surfaces, making pairing between thermally excited states above the lower Fermi surface and below the upper Fermi surface possible. As a consequence, as $T$ is increased from zero, there is a range of $T$ within which $\delta \mu_{1}$ is larger than at $T=0$. At still higher temperatures, of course, all pairing is lost.

In applying the work of Clogston and Chandrasekhar to color superconductivity, there have been two extensions to their analysis. First, recall that only two colors of up and down quarks pair. In describing the mixed phase associated with the first order phase transition, one must take careful account of the unpaired blue quarks. This has been done by Bedaque [11]. Second, in Refs. [9, 10] the transition between the color-flavor locked phase and the two-flavor color superconducting phase has been studied, under the assumption that $\mu_{u}=\mu_{d}=\mu_{s}$ but with $p_{F}^{s} \neq p_{F}^{u, d}$ because of the nonzero strange quark mass. The first order transition that these authors describe is similar to that of Clogston and Chandrasekhar, as it is associated with the unpairing of us and $u d$ Cooper pairs, but it differs in that the analogue of the normal state is one in which $u$ and $d$ quarks remain paired. As we have seen above, treating a realistic situation requires relaxing the assumption of equal chemical potentials.

\subsection{Non-isotropic (LOFF) pairing}

The Clogston and Chandrasekhar analysis of the first order unpairing transition assumes that the only possible phases of the system are a BCS phase and the normal phase. However, there is good reason to think that another state can occur in the crossover region between BCS and no pairing. As was first realized by Larkin and Ovchinnikov [12 and Fulde and Ferrell 13 (LOFF), whereas the BCS state requires pairing between fermions with equal and opposite momenta, when $\delta \mu \sim \delta \mu_{1}$ it may be more favorable to form a condensate of Cooper pairs with nonzero total momentum. By pairing quarks with momenta which are not equal and opposite, some Cooper pairs are allowed to have both the up and the down quarks on their respective Fermi surfaces even when $\delta \mu \neq 0$. LOFF found that within a range of $\delta \mu$ near $\delta \mu_{1}$, a condensate of Cooper pairs with momenta $\mathbf{q}+\mathbf{p}$ and $\mathbf{q}-\mathbf{p}$ (see Figure 1) is favored over either the BCS condensate or the normal state. Here, our notation is such that $\mathbf{p}$ specifies a particular Cooper pair, while $\mathbf{q}$ is a fixed vector, the same for 
all pairs, which characterizes a given LOFF state. The magnitude $|\mathbf{q}|$ is determined by minimizing the free energy; the direction of $\mathbf{q}$ is chosen spontaneously. The resulting LOFF state breaks translational and rotational invariance. In position space, it describes a condensate which varies as a plane wave with wave vector $2 \mathbf{q}$.

Once one has demonstrated an instability to the formation of a plane wave, it is natural to expect that the state which actually develops has a crystalline structure. Larkin and Ovchinnikov in fact argue that the favored configuration is a crystalline condensate which varies in space like a one-dimensional standing wave, $\cos (2 \mathbf{q} \cdot \mathbf{r})$. Such a condensate vanishes along nodal planes 12. Subsequent analyses suggest that the crystal structure may be more complicated. Shimahara [41] has shown that in two dimensions, the LOFF state favors different crystal structures at different temperatures: a hexagonal crystal at low temperatures, square at higher temperatures, then a triangular crystal and finally a one-dimensional standing wave as Larkin and Ovchinnikov suggested at temperatures that are higher still. In three dimensions, the question of which crystal structure is favored seems unresolved 42.

LOFF did their analysis in the same context as that of Clogston and Chandrasekhar: electromagnetic superconductivity in a magnetic field which causes a Zeeman splitting while not inducing screening currents. They were seeking to model the physics of magnetic impurities in a superconductor. Magnetic effects on the motion of the electrons 43 and the scattering of electrons off non-magnetic impurities [44, 45] disfavor the LOFF state. Although signs of the BCS to LOFF transition in the heavy fermion superconductor $\mathrm{UPd}_{2} \mathrm{Al}_{3}$ have been reported 46, the interpretation of these experiments is not unambiguous [47. It has also been suggested that the LOFF phase may be more easily realized in condensed matter systems which are two-dimensional 41, 48 or one-dimensional 49, both because in these cases $\delta \mu_{2}$ is larger than in three-dimensional systems and because the magnetic field applied precisely parallel to a one- or two-dimensional system does not affect the motion of electrons therein. Evidence for a LOFF phase in a quasi-two-dimensional layered organic superconductor has recently been reported 50.

None of the difficulties which have beset attempts to realize the LOFF phase in a system of electrons in a magnetic field arise in the QCD context of interest to us. Differences between quark chemical potentials are generic and the physics which leads to these differences has nothing to do with the motion of the quarks. We therefore expect the original analysis of LOFF (without the later complications added in order to treat the difficulties in the condensed matter physics context) to be a good starting point. In this paper we use an analysis based on that originally done by Fulde and Ferrell [13], but described in more detail by Takada and Izuyama [51], to argue that for appropriate values of quark number densities, the color superconducting gap may vary periodically in space, forming a crystalline pattern. More precisely, what we will demonstrate is that if some difference between chemical potentials falls in the appropriate range, quark matter is unstable to the spontaneous breaking of translational invariance by the formation of condensates 


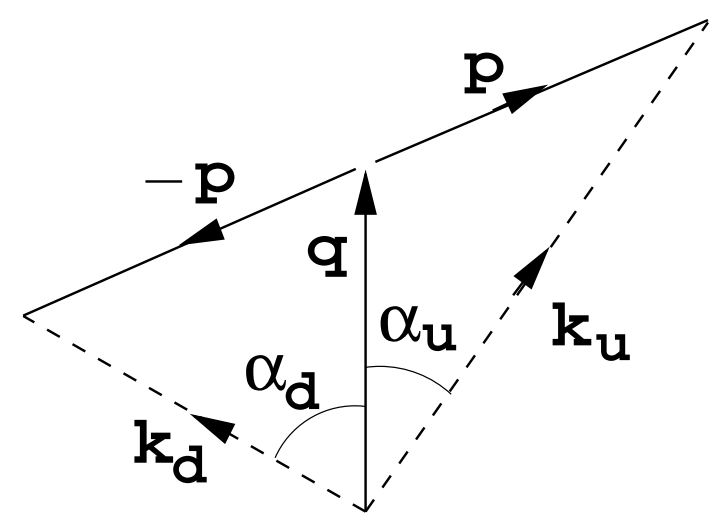

Figure 1: The momenta $\mathbf{k}_{u}$ and $\mathbf{k}_{d}$ of the two members of a LOFF-state Cooper pair. We choose the vector $\mathbf{q}$, common to all Cooper pairs, to coincide with the $z$-axis. The angles $\alpha_{u}(\mathbf{p})$ and $\alpha_{d}(\mathbf{p})$ indicate the polar angles of $\mathbf{k}_{u}$ and $\mathbf{k}_{d}$, respectively.

which vary in space like a plane wave. Following Larkin and Ovchinnikov [12, we expect that once there is an instability to the formation of plane waves the condensate that results will be crystalline, but we leave the determination of the crystal structure of the condensate to future work.

\section{The LOFF state}

We begin our analysis of a LOFF state for quark matter by constructing a variational ansatz for the LOFF wavefunction. As motivated by the preceding discussion, we consider Cooper pairs which consist of an up quark and a down quark with respective momenta

$$
\mathbf{k}_{u}=\mathbf{q}+\mathbf{p}, \quad \mathbf{k}_{d}=\mathbf{q}-\mathbf{p}
$$

so that $\mathbf{p}$ identifies a particular quark pair, and every quark pair in the condensate has the same nonzero total momentum 2q. This arrangement is shown in Figure 1 . The helicity and color structure are obtained by analogy with the "2SC" state as described in previous work [3, 4]: the quark pairs will be color $\overline{\mathbf{3}}$ antitriplets, and in our ansatz we consider only pairing between quarks of the same helicity.

With this in mind, here is a suitable trial wavefunction for the LOFF state with 
wavevector $\mathbf{q}[12$, 13, 51]:

$$
\begin{aligned}
\left|\Psi_{\mathbf{q}}\right\rangle & =B_{L}^{\dagger} B_{R}^{\dagger}|0\rangle, \\
B_{L}^{\dagger} & =\prod_{\mathbf{p} \in \mathcal{P}, \alpha, \beta}\left(\cos \theta_{L}(\mathbf{p})+\epsilon^{\alpha \beta 3} \mathrm{e}^{i \xi_{L}(\mathbf{p})} \sin \theta_{L}(\mathbf{p}) a_{L u \alpha}^{\dagger}(\mathbf{q}+\mathbf{p}) a_{L d \beta}^{\dagger}(\mathbf{q}-\mathbf{p})\right) \\
& \times \prod_{\mathbf{p} \in \mathcal{B}_{u}, \alpha} a_{L u \alpha}^{\dagger}(\mathbf{q}+\mathbf{p}) \times \prod_{\mathbf{p} \in \mathcal{B}_{d}, \beta} a_{L d \beta}^{\dagger}(\mathbf{q}-\mathbf{p}), \\
B_{R}^{\dagger} & =\text { as above, } L \rightarrow R,
\end{aligned}
$$

where $\alpha, \beta$ are color indices, $u, d$ and $L, R$ are the usual flavor and helicity labels, and $a^{\dagger}$ is the particle creation operator (for example, $a_{L d \alpha}^{\dagger}$ creates a left-handed down quark with color $\alpha$ ). The $\theta$ 's and $\xi$ 's are the variational parameters of our ansatz: they are to be chosen to minimize the free energy of the LOFF state, as described in the next section. The first product in equation (3.2) creates quark pairs within a restricted region $\mathcal{P}$ of the total phase space. This allowed "pairing region" will be discussed below. The next product fills a "blocking region" $\mathcal{B}_{u}$ with unpaired up quarks: these are up quarks with momenta $\mathbf{q}+\mathbf{p}$ for which there are no corresponding down quarks with momenta $\mathbf{q}-\mathbf{p}$. The final product fills the blocking region $\mathcal{B}_{d}$ with unpaired down quarks. The ansatz does not contain a term that would create antiparticle pairs: we have checked the effect of such a term and found that it has no qualitative effect on our results.

To complete the specification of our ansatz we need to describe the allowed pairing and blocking regions in phase space. These regions are largely determined by Pauli blocking as a result of populated Fermi seas. In the absence of pairing interactions, the system is in the "normal" state and up and down quarks are distributed in Fermi seas with Fermi momenta $p_{F}^{u}=\mu_{u}$ and $p_{F}^{d}=\mu_{d}$, respectively (recall that we consider massless quarks only, so the single particle energy of a quark with momentum $\mathbf{k}$ is $\epsilon(\mathbf{k})=|\mathbf{k}|)$. An up quark carries momentum $\mathbf{k}_{u}=\mathbf{p}+\mathbf{q}$; in $\mathbf{p}$-space, therefore, the Fermi sea of up quarks corresponds to a sphere of radius $\mu_{u}=\bar{\mu}-\delta \mu$ centered at $-\mathbf{q}$. Similarly, a down quark carries momentum $\mathbf{k}_{d}=-\mathbf{p}+\mathbf{q}$, giving a sphere in $\mathbf{p}$-space of radius $\mu_{d}=\bar{\mu}+\delta \mu$ centered at $+\mathbf{q}$. The two offset spheres are shown in Figure 22 a (we have drawn the case $|\mathbf{q}|>\delta \mu$ so that the two Fermi surfaces intersect in $\mathbf{p}$-space). In the limit of arbitrarily weak interactions, the blocking region $\mathcal{B}_{u}$ corresponds to the lower shaded area in the figure: pairing does not occur here since the region is inside the Fermi sea of up quarks, but outside the Fermi sea of down quarks. Similarly the upper shaded area is the blocking region $\mathcal{B}_{d}$. The entire unshaded area is the pairing region $\mathcal{P}$ : it includes the region inside both spheres, where hole-hole pairing can occur, and the region outside both spheres, where particle-particle pairing can occur.

We can now explain how the LOFF wavefunction ansatz can describe the normal state with no condensate: we choose $\theta_{L}(\mathbf{p})=\theta_{R}(\mathbf{p})=\pi / 2$ for $\mathbf{p}$ inside both Fermi spheres, and otherwise all the $\theta$ 's are zero. With this choice the first term in 


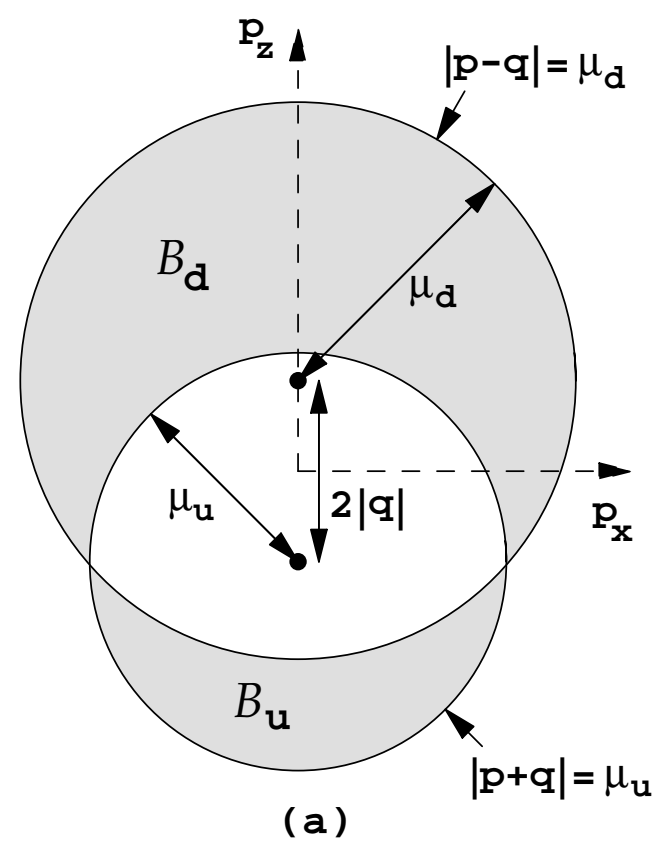

(a)

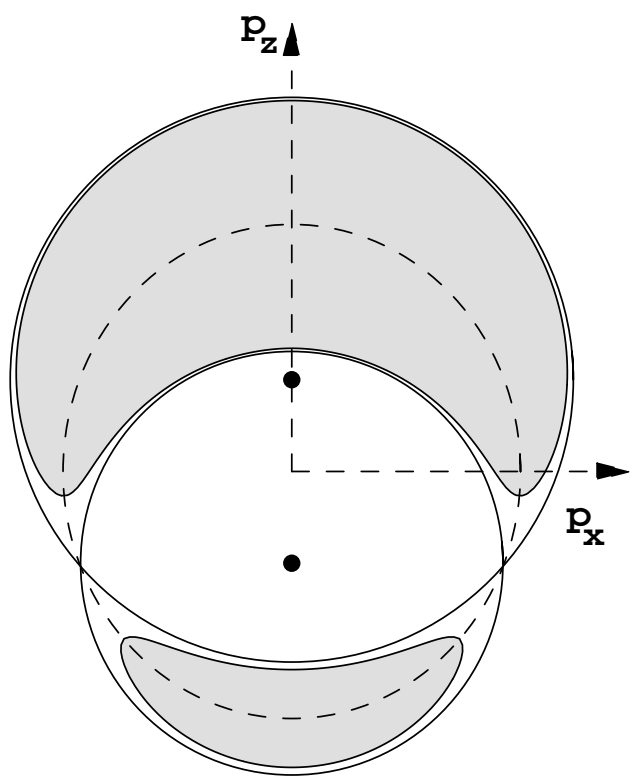

(b)

Figure 2: The LOFF phase space, as a function of $\mathbf{p}$ (Eq. (3.1)). We show the $p_{y}=0$ plane. (a) The phase space in the limit of arbitrarily weak interactions. In the shaded blocking regions $\mathcal{B}_{u}$ and $\mathcal{B}_{d}$, no pairing is possible. In the inner unshaded region, an interaction can induce hole-hole pairs. In the outer unshaded region, an interaction can induce particle-particle pairs. The region $\mathcal{P}($ Eq. (3.2)) is the whole unshaded area. (b) When the effects of interactions and the formation of the LOFF state are taken into account, the blocking regions shrink. The BCS singularity occurs on the dashed ellipse, defined by $\epsilon_{u}+\epsilon_{d}=\mu_{u}+\mu_{d}$, where making a Cooper pair costs no free energy in the free case.

Eq. (3.2) fills that part of each Fermi sea corresponding to the inner unshaded region of Figure 2a. The $\mathcal{B}_{u}$ and $\mathcal{B}_{d}$ terms fill out the remainder of each Fermi sea to obtain the normal state. Note that in the absence of pairing, the normal state can be described with any choice of $\mathbf{q}$. The most convenient choice is $\mathbf{q}=0$, in which case $\mathbf{k}_{u}=\mathbf{k}_{d}=\mathbf{p}, \mathcal{B}_{u}$ vanishes, and $\mathcal{B}_{d}$ is a spherical shell. Other choices of $\mathbf{q}$ correspond to choosing different origins of $\mathbf{k}_{u}$-space and $\mathbf{k}_{d}$-space, but in the absence of any interactions this has no consequence. Once we turn on interactions and allow pairing, we expect a particular $|\mathbf{q}|$ to be favored.

The phase space picture changes slightly when pairing interactions are included: the blocking regions are smaller when a LOFF condensate is present, as indicated in Figure 2 $2 \mathrm{~b}$. We will account for this effect in the next section. With smaller blocking regions, a larger portion of the phase space becomes available for LOFF 
pairing. Such pairing is guaranteed to be energetically favorable when it costs zero free energy to create an up quark and a down quark, since these quarks can then pair to obtain a negative interaction energy. The zero free energy condition is

$$
\epsilon\left(\mathbf{k}_{u}\right)+\epsilon\left(\mathbf{k}_{d}\right)=\mu_{u}+\mu_{d}=2 \bar{\mu}
$$

where $\epsilon(\mathbf{k})$ is the single particle energy of a quark with momentum k. For massless quarks, we obtain $|\mathbf{q}+\mathbf{p}|+|\mathbf{q}-\mathbf{p}|=2 \bar{\mu}$, which describes an ellipsoidal surface in p-space. This surface is indicated by the ellipse shown in Figure $2 \mathrm{~b}$; notice that the ellipsoid and the two Fermi surfaces all intersect at a circle.

If the interaction is weak, we expect LOFF pairing to be favored in a thin layer of phase space around this ellipsoid. This is manifest in the gap equation derived in the next section (Eq. (4.9)) in which, as in BCS theory, we find a divergent integrand on this ellipsoid in the absence of pairing. Pairing smoothes the divergence. As the interaction gets stronger, the layer of favored pairing gets thicker. If there were no blocking regions, we could use the entire ellipsoid, just as BCS pairs condense over the entire spherical surface $|\mathbf{p}|=\mu$ in the symmetric, $\delta \mu=|\mathbf{q}|=0$ case. However, as shown in Figure $2 \mathrm{~b}$, the blocking regions exclude pairing over most of the ellipsoid, leaving a ribbon of unsuppressed LOFF pairing in the vicinity of the circle where the Fermi surfaces intersect. This agrees with our expectation for the particle distribution in the LOFF state: it is as in the normal state, except that there is a restricted region (around the aforementioned ribbon) where each quark in a pair can be near its Fermi surface.

Although the constant single-particle energy contours for noninteracting up and down quarks cross in $\mathbf{p}$-space (see Figure 2a), we emphasize that the Fermi surfaces of up and down quarks do not cross in momentum $\left(\mathbf{k}_{u^{-}}\right.$and $\left.\mathbf{k}_{d^{-}}\right)$space. The $\mathbf{p}$-space ribbon of unsuppressed pairing corresponds to unsuppressed pairing between up and down quarks with momenta around $\mathbf{k}$-space ribbons near their respective (disjoint) Fermi surfaces.

In the limit of arbitrarily weak interactions, the ribbon in momentum space along which pairing is unsuppressed shrinks, as the blocking regions grow to exclude all of the ellipsoid except the one-dimensional circle at which the two spheres in Figure 8 intersect. This circle has insufficient phase space to lead to a singularity in the gap equation: the integrand is singular on this circle, but the integral does not diverge. Therefore, the LOFF state is not guaranteed to occur if one takes the weak coupling limit at fixed $\delta \mu$. In this respect, the LOFF state is like the BCS state at nonzero $\delta \mu$ : for weak coupling, $\Delta_{0} \rightarrow 0$ and because the BCS state can only exist if it has $\Delta_{0}>\sqrt{2} \delta \mu$, it must vanish for couplings weaker than some threshold. We shall see, however, that at any fixed weak coupling, the LOFF state, like the BCS state, is guaranteed to occur at some $\delta \mu$ : the BCS state arises if $\delta \mu<\delta \mu_{1}$ and the LOFF state arises if $\delta \mu_{1}<\delta \mu<\delta \mu_{2}$.

One of the most striking features of the LOFF state is the spin structure of the condensate. The familiar "2SC" state pairs quarks of the same helicity and 
opposite momentum, so the spins are antiparallel and the quarks are arranged in an antisymmetric combination to form spin singlet Cooper pairs. The LOFF state also pairs quarks of the same helicity, but now the quark momenta are no longer antiparallel, as can be seen from Figure 1. Therefore the LOFF Cooper pairs cannot be spin singlets: they are superpositions of both spin zero and spin one. This is revealed explicitly by evaluating the nonzero $\langle\psi \psi\rangle$ expectation values in the LOFF state:

$$
\begin{aligned}
-\left\langle\Psi_{\mathbf{q}}\left|\epsilon_{i j} \epsilon_{\alpha \beta 3} \psi^{i \alpha}(\mathbf{r}) C L \psi^{j \beta}(\mathbf{r})\right| \Psi_{\mathbf{q}}\right\rangle & =2 \Gamma_{A}^{L} \mathrm{e}^{i 2 \mathbf{q} \cdot \mathbf{r}} \\
i\left\langle\Psi_{\mathbf{q}}\left|\left(\sigma_{1}\right)_{i j} \epsilon_{\alpha \beta 3} \psi^{i \alpha}(\mathbf{r}) C L \sigma^{03} \psi^{j \beta}(\mathbf{r})\right| \Psi_{\mathbf{q}}\right\rangle & =2 \Gamma_{B}^{L} \mathrm{e}^{i 2 \mathbf{q} \cdot \mathbf{r}}
\end{aligned}
$$

where $i, j$ are flavor indices $\left(1=\right.$ up, $2=$ down), $\alpha, \beta$ are color indices, $C=i \gamma^{0} \gamma^{2}$, $L=\left(1-\gamma_{5}\right) / 2$ is the usual left-handed projection operator, and $\sigma_{\mu \nu}=(i / 2)\left[\gamma_{\mu}, \gamma_{\nu}\right]$. The constants $\Gamma_{A}^{L}$ and $\Gamma_{B}^{L}$ are left-handed $J=0$ and $J=1$ condensates, respectively. $\Gamma_{A}^{R}$ and $\Gamma_{B}^{R}$ are defined analogously. The $\Gamma^{\prime}$ 's can be expressed in terms of the variational parameters of the $\mathrm{LOFF}$ wavefunction:

$$
\begin{aligned}
\Gamma_{A}^{L} & =\frac{4}{V} \sum_{\mathbf{p} \in \mathcal{P}} \sin \theta_{L}(\mathbf{p}) \cos \theta_{L}(\mathbf{p}) \mathrm{e}^{i \xi_{L}(\mathbf{p})} \sin \left(\frac{\alpha_{u}(\mathbf{p})+\alpha_{d}(\mathbf{p})}{2}\right) \mathrm{e}^{-i \phi(\mathbf{p})} \\
\Gamma_{B}^{L} & =\frac{4}{V} \sum_{\mathbf{p} \in \mathcal{P}} \sin \theta_{L}(\mathbf{p}) \cos \theta_{L}(\mathbf{p}) \mathrm{e}^{i \xi_{L}(\mathbf{p})} \sin \left(\frac{\alpha_{u}(\mathbf{p})-\alpha_{d}(\mathbf{p})}{2}\right) \mathrm{e}^{-i \phi(\mathbf{p})}
\end{aligned}
$$

Here $V$ is the spatial volume of the system, $\alpha(\mathbf{p})$ are the polar angles of the quark momenta, as in Figure 1, and the dependence on the azimuthal angle $\phi$ follows from our use of the spinor conventions described in Refs. [2, 3, 6]. The expressions for $\Gamma_{A}^{R}$ and $\Gamma_{B}^{R}$ are the same as those in (3.5) except that $\phi(\mathbf{p})$ is replaced by $\pi-\phi(\mathbf{p})$. In Eq. (3.5) and throughout, $(1 / V) \sum_{\mathbf{p}}$ becomes $\int d^{3} p /(2 \pi)^{3}$ in an infinite system.

Once we have derived a gap equation by minimizing the free energy with respect to these variational parameters, we expect the condensates to be simply related to gap parameters occurring in the gap equation. We will see explicitly how $\Gamma_{A}$ and $\Gamma_{B}$ are determined in the next section.

Notice that the condensates of Eq. (3.4) are plane waves in position space by virtue of the nonzero momentum $2 \mathbf{q}$ of a Cooper pair. $\Gamma_{A}$ describes pairing which is antisymmetric in color, spin, and flavor, while $\Gamma_{B}$ describes pairing which is antisymmetric in color but symmetric in spin and flavor (in each case, Pauli statistics are obeyed). In the original LOFF condensate of electrons there can be no $\Gamma_{B}$, since electrons have no color or flavor, so that only the spin antisymmetric pairing is possible.

The $J=0$ condensates $\langle\psi C L \psi\rangle,\langle\psi C R \psi\rangle$ are Lorentz scalars (mixed under parity), while the $J=1$ condensates $\left\langle\psi C L \sigma^{03} \psi\right\rangle,\left\langle\psi C R \sigma^{03} \psi\right\rangle$ are 3 -vectors (also mixed under parity) which point in the $z$-direction, parallel to the spontaneously chosen direction $\hat{\mathbf{q}}$ of the LOFF state. Because the ansatz contains a $J=1$ component, it would be interesting to generalize it to include the possibility of $L R$ pairing, in addition to $L L$ and $R R$ pairing. We discuss this further in Section 6 . 
The possibility of a LOFF phase in QCD has been mentioned briefly in a different context. In their analysis of quark matter with a very large isospin density (with large Fermi momenta for down and anti-up quarks) Son and Stephanov have noted that if the $d$ and $\bar{u}$ Fermi momenta differ suitably, a LOFF phase will arise [52].

In the physically realizable context of large baryon number density, pairing between quarks and holes with nonzero total momentum has also been discussed [53, 54, 55, 56. This results in a condensate with the quantum numbers of $\langle\bar{q} q\rangle$, which varies in space with a wave number equal to $2 \mu$; in contrast, the LOFF phase describes a diquark condensate which varies with a wave number $2|\mathbf{q}|$ comparable to $2 \delta \mu$. The crystalline chiral condensate [56] is favored in QCD at asymptotically high densities only if the number of colors is very large [53], greater than about $N_{c}=1000$ [54, 55]. It may arise at lower densities in QCD with fewer colors, but apparently not in QCD with $N_{c}=3$ 56. Note that crystalline color superconductivity is guaranteed to occur at arbitrarily weak coupling for suitably chosen $\delta \mu$, while a crystalline chiral condensate cannot form anywhere in the phase diagram if the coupling is weak.

\section{The gap equation and free energy}

Having presented a trial wavefunction for the LOFF state, we now proceed to minimize the expectation value of the free energy $\langle F\rangle$ with respect to the variational parameters of the wavefunction (the $\theta$ 's and $\xi$ 's of equation (B.2) ) to obtain a LOFF gap equation. The free energy is $F=H-\mu_{u} N_{u}-\mu_{d} N_{d}$, where $H$ is the Hamiltonian, and $N_{u}$ and $N_{d}$ are the number operators for up and down quarks, respectively. We choose a model Hamiltonian which has a free quark term $H_{0}$ and an interaction term $H_{I}$, and write the free energy as $F=F_{0}+H_{I}$, where $F_{0}=H_{0}-\mu_{u} N_{u}-\mu_{d} N_{d}$ is the free energy for noninteracting quarks. To describe the pairing interaction between quarks, we use an NJL model consisting of a four-fermion interaction with the color and flavor structure of one-gluon exchange:

$$
H_{I}=\frac{3}{8} \int d^{3} x\left[G_{E}\left(\bar{\psi} \gamma^{0} T^{A} \psi\right)\left(\bar{\psi} \gamma^{0} T^{A} \psi\right)-G_{M}\left(\bar{\psi} \gamma^{i} T^{A} \psi\right)\left(\bar{\psi} \gamma^{i} T^{A} \psi\right)\right]
$$

where the $T^{A}$ are the color $S U(3)$ generators, normalized so that $\operatorname{tr}\left(T^{A} T^{B}\right)=2 \delta^{A B}$. Notice that we have relaxed some constraints on the spin structure of one-gluon exchange: we allow for the possibility of independent couplings $G_{E}$ and $G_{M}$ for electric and magnetic gluons, respectively. This spoils Lorentz boost invariance but there is no reason to insist on boost invariance in a finite-density system. Indeed, in high density quark matter we expect screening of electric gluons but only Landau damping of magnetic gluons, and we might choose to model these effects by setting $G_{E} \ll G_{M}$. We postpone a discussion of these issues and their implications for the LOFF state until Section 6. For now, we restrict ourselves to the case of Lorentz invariant single gluon exchange, by letting $G_{E}=G_{M}=G>0$. 
We need to evaluate the expectation value of $F$ in the LOFF state to obtain an expression for the free energy of the system in terms of the variational parameters of the ansatz. The noninteracting part of the free energy is simply

$$
\begin{aligned}
\left\langle F_{0}\right\rangle= & \sum_{\mathbf{p} \in \mathcal{B}_{u}} 2\left(|\mathbf{q}+\mathbf{p}|-\mu_{u}\right)+\sum_{\mathbf{p} \in \mathcal{B}_{d}} 2\left(|\mathbf{q}-\mathbf{p}|-\mu_{d}\right) \\
& +\sum_{\mathbf{p} \in \mathcal{P}} 2\left(|\mathbf{q}+\mathbf{p}|+|\mathbf{q}-\mathbf{p}|-\mu_{u}-\mu_{d}\right) \sin ^{2} \theta_{L}(\mathbf{p}) \\
& +(\text { same, with } L \rightarrow R) .
\end{aligned}
$$

The first and second terms represent the contributions of the unpaired left-handed up and down quarks, respectively. The third term gives the (noninteracting) free energy of the left-handed quark pairs. The three terms are all repeated with $L$ replaced by $R$ to include the free energy for the right-handed quarks. The factors of two in equation (4.2) appear because there are two quark colors ("red" and "green") involved in the the condensate. The "blue" quarks do not participate in the pairing interaction and instead behave as free particles: the blue up and down quarks fill Fermi seas with Fermi momenta $p_{F}^{u}=\mu_{u}$ and $p_{F}^{d}=\mu_{d}$, respectively. Below, we will want to compare the free energy of the LOFF, BCS and normal states. Since at any given $\mu_{u}$ and $\mu_{d}$ the free energy of the spectator quarks is the same in all three states, we can neglect these blue quarks in the remainder of our analysis even though they do contribute to the total free energy.

The expectation value of $H_{I}$ gives the total binding energy of the pairing interaction:

$$
\left\langle H_{I}\right\rangle=-\frac{1}{2} G V\left(\left|\Gamma_{A}^{L}\right|^{2}+\left|\Gamma_{A}^{R}\right|^{2}\right)
$$

where the $\Gamma_{A}$ 's are the $J=0 \mathrm{LOFF}$ condensates defined in equations (3.5). These condensates are simply related to $J=0$ LOFF gap parameters defined as

$$
\Delta_{A}^{\{L, R\}}=G \Gamma_{A}^{\{L, R\}}
$$

The gap parameters $\Delta_{A}$ correspond to 1PI Green's functions and are the quantities which will appear in the quasiparticle dispersion relations and for which we will derive the self-consistency conditions conventionally called gap equations. We see from Eq. (4.3) that with $G>0$ the interaction is attractive in the $J=0$ channel and is neither attractive nor repulsive in the $J=1$ channel.

Our ansatz breaks rotational invariance, so once $J=0$ pairing occurs $\left(\Gamma_{A} \neq 0\right)$ we expect that there will also be $J=1$ pairing $\left(\Gamma_{B} \neq 0\right)$. As we have seen, this arises even in the absence of any interaction in the $J=1$ channel as a consequence of the fact that the momenta of two quarks in a Cooper pair are not anti-parallel if $\mathbf{q} \neq 0$. Because $\langle H\rangle$ is independent of $\Gamma_{B}$, the quasiparticle dispersion relations must also be independent of $\Gamma_{B}$. That is, the $J=1$ gap parameter must vanish: $\Delta_{B}=0$. In Section 6, we shall see by direct calculation that $\Delta_{B}$ is proportional to $\left(G_{E}-G_{M}\right) \Gamma_{B}$. In the present analysis with $G_{E}=G_{M}$, therefore, $\Delta_{B}=0$ while $\Gamma_{B} \neq 0$. 
The $\xi$ 's are chosen to cancel the azimuthal phases $\phi(\mathbf{p})$ in equations (3.5). By this choice we obtain maximum coherence in the sums over $\mathbf{p}$, giving the largest possible magnitudes for the condensates and gap parameters. We have

$$
\xi_{L}(\mathbf{p})=\phi(\mathbf{p})+\varphi_{L}, \quad \xi_{R}(\mathbf{p})=\pi-\phi(\mathbf{p})+\varphi_{R}
$$

where $\varphi_{L}$ and $\varphi_{R}$ are arbitrary p-independent angles. These constant phases do not affect the free energy - they correspond to the Goldstone bosons for the broken left-handed and right-handed baryon number symmetries - and are therefore not constrained by the variational procedure. For convenience, we set $\varphi_{L}=\varphi_{R}=0$ and obtain condensates and gap parameters that are purely real.

The relative phase $\varphi_{L}-\varphi_{R}$ determines how the LOFF condensate transforms under a parity transformation. Its value determines whether the $J=0$ condensate is scalar, pseudoscalar, or an arbitrary combination of the two and whether the $J=1$ condensate is vector, pseudovector, or an arbitrary combination. Because single gluon exchange cannot change the handedness of a massless quark, the leftand right-handed condensates in the LOFF phase are not coupled in the free energy of Eq. (4.3.) Our choice of interaction Hamiltonian therefore allows an arbitrary choice of $\varphi_{L}-\varphi_{R}$. A global $U(1)_{A}$ transformation changes $\varphi_{L}-\varphi_{R}$, and indeed this is a symmetry of our toy model. If we included $U(1)_{A}$-breaking interactions in our Hamiltonian, to obtain a more complete description of QCD, we would find that the free energy depends on $\varphi_{L}-\varphi_{R}$, and thus selects a preferred value. For example, had we taken $H_{I}$ to be the two-flavor instanton interaction as in Ref. [3, (4), the interaction energy would appear as $\Gamma^{L *} \Gamma^{R}+\Gamma^{L} \Gamma^{R *}$ instead of as in (4.3). This would enforce a fixed phase relation $\varphi_{L}-\varphi_{R}=0$, favoring condensates which are parity conserving [3, [4].

We now apply the variational method to determine the angles $\theta(\mathbf{p})$ in our trial wavefunction, by requiring that the free energy is minimized: $\partial\langle F\rangle / \partial \theta(\mathbf{p})=0$. This is complicated by the fact that the pairing region $\mathcal{P}$ and the blocking regions $\mathcal{B}_{u}$ and $\mathcal{B}_{d}$ are themselves implicitly dependent on the $\theta$ angles: these angles determine the extent of the LOFF pairing, and the phase space regions $\mathcal{P}, \mathcal{B}_{u}$ and $\mathcal{B}_{d}$ change when a condensate is present, as mentioned in Section 3. For now we simply ignore any $\theta$-dependence of the phase space regions; our result will nevertheless turn out to be correct. Everything is the same for left and right condensates so we hereafter drop the $L$ and $R$ labels. Upon variation with respect to $\theta(\mathbf{p})$, we obtain

$$
\tan 2 \theta(\mathbf{p})=\frac{2 \Delta_{A} \sin \left(\beta_{A}(\mathbf{p}) / 2\right)}{|\mathbf{q}+\mathbf{p}|+|\mathbf{q}-\mathbf{p}|-\mu_{u}-\mu_{d}}
$$

where $\beta_{A}(\mathbf{p})=\alpha_{u}(\mathbf{p})+\alpha_{d}(\mathbf{p})$ is the angle between the two quark momenta in a LOFF pair, as shown in Figure [1. Notice that the denominator on the right hand side of the above expression vanishes along the ellipsoidal surface of optimal LOFF pairing described in Section 3 . When $\mathbf{q}=0$, the quark momenta are antiparallel so $\beta_{A}(\mathbf{p})=\pi$ and Eq. (4.6) reduces to the simple BCS result: $\tan 2 \theta=\Delta_{A} /(|\mathbf{p}|-\bar{\mu})$. 
With the $\theta$ angles now expressed in terms of a gap parameter $\Delta_{A}$, we turn to the LOFF quasiparticle dispersion relations. They can be obtained by taking the absolute value of the expressions

$$
\begin{aligned}
E_{1}(\mathbf{p})=\delta \mu & +\frac{1}{2}(|\mathbf{q}+\mathbf{p}|-|\mathbf{q}-\mathbf{p}|) \\
& +\frac{1}{2} \sqrt{(|\mathbf{q}+\mathbf{p}|+|\mathbf{q}-\mathbf{p}|-2 \bar{\mu})^{2}+4 \Delta_{A}^{2} \sin ^{2}\left(\frac{1}{2} \beta_{A}(\mathbf{p})\right)} \\
E_{2}(\mathbf{p})=-\delta \mu & -\frac{1}{2}(|\mathbf{q}+\mathbf{p}|-|\mathbf{q}-\mathbf{p}|) \\
& +\frac{1}{2} \sqrt{(|\mathbf{q}+\mathbf{p}|+|\mathbf{q}-\mathbf{p}|-2 \bar{\mu})^{2}+4 \Delta_{A}^{2} \sin ^{2}\left(\frac{1}{2} \beta_{A}(\mathbf{p})\right)}
\end{aligned}
$$

whose meaning we now describe. For regions of $\mathbf{p}$-space which are well outside both Fermi surfaces, $E_{1}\left(E_{2}\right)$ is the free energy cost of removing a LOFF pair and adding an up quark with momentum $\mathbf{q}+\mathbf{p}$ (a down quark with momentum $\mathbf{q}-\mathbf{p}$ ). For regions of $\mathbf{p}$-space which are well inside both Fermi surfaces, $E_{1}\left(E_{2}\right)$ is the free energy cost of removing a LOFF hole pair and adding a down hole with momentum $\mathbf{q}-\mathbf{p}$ (an up hole with momentum $\mathbf{q}+\mathbf{p}$ ). Where the Fermi surfaces cross in $\mathbf{p}-$ space and pairing is maximal, both quasiparticles are equal superpositions of up and down. In the region of $\mathbf{p}$-space which is well inside the up Fermi surface but well outside the down Fermi surface, $E_{1}$ is negative, corresponding to a domain in which it is energetically favorable to have an unpaired up quark with momentum $\mathbf{q}+\mathbf{p}$ rather than a $(\mathbf{q}+\mathbf{p}, \mathbf{q}-\mathbf{p})$ quark pair. Similarly, $E_{2}$ is negative where it is favorable to have an unpaired down quark with momentum $\mathbf{q}-\mathbf{p}$ rather than a LOFF pair. Equations (4.7) allow us to finally complete our description of the LOFF phase by specifying the definitions of the phase space regions $\mathcal{P}, \mathcal{B}_{u}$ and $\mathcal{B}_{d}$. The blocking region $\mathcal{B}_{u}$ is the region where $E_{1}(\mathbf{p})$ is negative, and unpaired up quarks are favored over LOFF pairs. Similarly $\mathcal{B}_{d}$ is the region where $E_{2}(\mathbf{p})$ is negative. The regions $E_{1}<0$ and $E_{2}<0$ are shown as the shaded areas in Figure 2a for $\Delta_{A}=0$, and in Figure 2 $2 \mathrm{~b}$ for $\Delta_{A} \neq 0$. LOFF pairing occurs in the region where $E_{1}$ and $E_{2}$ are both positive:

$$
\mathcal{P}=\left\{\mathbf{p} \mid E_{1}(\mathbf{p})>0 \text { and } E_{2}(\mathbf{p})>0\right\}
$$

corresponding to the entire unshaded regions of Figure 2. The actual quasiparticle dispersion functions are $\left|E_{1}(\mathbf{p})\right|$ and $\left|E_{2}(\mathbf{p})\right|$ : they are nonnegative everywhere, since they represent energies of perturbations of the LOFF state which is the presumed ground state of the system. In the blocking regions, elementary excitations are created by replacing an unpaired quark with a quark pair, and vice versa in the pairing region. When $\mathbf{q}=0$, Eqs. (4.7) reduce to the more familiar BCS result: $E_{\{1,2\}}(\mathbf{p})= \pm \delta \mu+\sqrt{(|\mathbf{p}|-\bar{\mu})^{2}+\Delta_{A}^{2}}$.

With the boundaries of the blocking regions specified, one can verify by explicit calculation that the variation of these boundaries upon variation of the $\theta$ 's does not

${ }^{4}$ Since the LOFF condensate contains pairs with momentum $2 \mathbf{q}$, the momentum of its quasiparticle excitations is only defined modulo $2 \mathbf{q}$. The momentum, modulo $2 \mathbf{q}$, of a quasiparticle of energy $\left|E_{1}(\mathbf{p})\right|$ is $\mathbf{p} \bmod 2 \mathbf{q}$. 
change the free energy. This can be understood as follows. Notice that because we can create zero-energy quasiparticles on the boundaries of the blocking regions, there is no actual energy gap in the excitation spectrum of the LOFF state. The change in $\langle F\rangle$ due to variation of the boundaries of the blocking regions is zero because this variation simply creates zero-free-energy quasiparticles on these boundaries. This justifies our neglect of the $\theta$-dependence of the phase space regions in the derivation of Eq. (4.6).

Substituting the expression (4.6) for the $\theta$ angles into the expression (3.5) for the $\Gamma_{A}$ condensate, and using the relation $\Delta_{A}=G \Gamma_{A}$, we obtain a self-consistency equation for the gap parameter $\Delta_{A}$ :

$$
1=\frac{2 G}{V} \sum_{\mathbf{p} \in \mathcal{P}} \frac{2 \sin ^{2}\left(\frac{1}{2} \beta_{A}(\mathbf{p})\right)}{\sqrt{(|\mathbf{q}+\mathbf{p}|+|\mathbf{q}-\mathbf{p}|-2 \bar{\mu})^{2}+4 \Delta_{A}^{2} \sin ^{2}\left(\frac{1}{2} \beta_{A}(\mathbf{p})\right)}} .
$$

This can be compared to the BCS gap equation, obtained upon setting $\mathbf{q}=0$ and eliminating the blocking regions:

$$
1=\frac{2 G}{V} \sum_{\mathbf{p}} \frac{1}{\sqrt{(|\mathbf{p}|-\bar{\mu})^{2}+\Delta_{0}^{2}}} .
$$

Note that in the LOFF gap equation (4.9), the gap parameter appears on the right hand side both explicitly in the denominator and also implicitly in the definition of the pairing region $\mathcal{P}$, as given in (4.8). This means that if the $\mathbf{q} \rightarrow 0$ limit is taken at fixed $\delta \mu$, the LOFF gap equation will only become the BCS gap equation if the blocking regions vanish in this limit. This happens if, as $\mathbf{q} \rightarrow 0, \Delta_{A}$ tends to a limiting value which is greater than $\delta \mu$. A state with $\Delta_{A}<\delta \mu$ and $\mathbf{q}=0$ is "BCS-like", in that the Cooper pairs have zero momentum, but has no pairing within a region $p_{F}^{u}<|\mathbf{p}|<p_{F}^{d}$. Such states always have higher free energy than the BCS state obtained simply by solving the gap equation (4.10), appropriate if there are no blocking regions and $p_{F}^{u}=p_{F}^{d}$ [39].

In the next section we will solve the LOFF gap equation (4.9) and determine the circumstances in which the LOFF state is the true ground state of the system. Once we have obtained a solution to the gap equation (4.9) for $\Delta_{A}$, the condensates are given by $\Gamma_{A}=\Delta_{A} / G$ and

$$
\Gamma_{B}=\frac{2}{V} \sum_{\mathbf{p} \in \mathcal{P}} \frac{2 \Delta_{A} \sin \left(\frac{1}{2} \beta_{A}(\mathbf{p})\right) \sin \left(\frac{1}{2} \beta_{B}(\mathbf{p})\right)}{\sqrt{(|\mathbf{q}+\mathbf{p}|+|\mathbf{q}-\mathbf{p}|-2 \bar{\mu})^{2}+4 \Delta_{A}^{2} \sin ^{2}\left(\frac{1}{2} \beta_{A}(\mathbf{p})\right)}}
$$

where $\beta_{B}(\mathbf{p})=\alpha_{u}(\mathbf{p})-\alpha_{d}(\mathbf{p})$. (See Figure 1.) We now see explicitly that if the interaction is attractive in the $J=0$ channel, creating a nonzero $\Gamma_{A}$ and $\Delta_{A}$, a nonzero $J=1$ condensate $\Gamma_{B}$ is induced regardless of the fact that there is no interaction in the $J=1$ channel. As a check, note that if $\mathbf{q}=0, \sin \left(\frac{1}{2} \beta_{A}(\mathbf{p})\right)=1$ and $\sin \left(\frac{1}{2} \beta_{B}(\mathbf{p})\right)$ is given by the cosine of the polar angle of $\mathbf{p}$. The right hand side 
of (4.11) therefore vanishes upon integration, and $\Gamma_{B}$ vanishes when $\mathbf{q}=0$ as it should. It is now apparent that two features contribute to a nonzero $\Gamma_{B}$. The first is that the momenta in a quark pair are not antiparallel, which leads to the factors of $\sin \left(\frac{1}{2} \beta_{A}(\mathbf{p})\right)$ in Eq. (4.11). The second is that the pairing region is anisotropic,

since if it were not the factor of $\sin \left(\frac{1}{2} \beta_{B}(\mathbf{p})\right)$ would ensure that the right-hand side of (4.11) vanishes upon integration.

As written, the gap equations (4.9) and (4.10) are ultraviolet divergent. In QCD, of course, asymptotic freedom implies that the interaction between quarks decreases at large momentum transfer and we have not yet represented this fact in our toy model. In previous work [3, 6, 5], we chose to mimic the effects of asymptotic freedom (and to render the right hand side of the gap equation finite) by introducing a form factor associated with each fermion leg in the four-fermion interaction. This is not a good strategy when $\mathbf{q} \neq 0$. The two incident quarks carry momenta $\mathbf{q}+\mathbf{p}$ and $\mathbf{q}-\mathbf{p}$ while the outgoing quarks carry momenta $\mathbf{q}+\mathbf{p}^{\prime}$ and $\mathbf{q}-\mathbf{p}^{\prime}$. Were we to cut off these four momenta with form factors on each leg, we would have a cutoff which depends explicitly on $\mathbf{q}$. This is not a good representation of what happens in full QCD, in which the condition for when the interaction becomes weak is determined by the momentum $\mathbf{p}-\mathbf{p}^{\prime}$ transferred through the gluon and has nothing to do with q. For simplicity, we choose to introduce a hard cutoff in our NJL model, rather than a smooth form factor, and choose simply to cut off the momentum p. This is not equivalent to cutting off the momentum transfer, but has the desired feature of being a q-independent cutoff. That is, we limit the integration region to $|\mathbf{p}|<\Lambda$ in the BCS gap equation (4.10) and to $\{\mathbf{p} \in \mathcal{P}$ and $|\mathbf{p}|<\Lambda\}$ in the LOFF gap equation (4.9). In the BCS case, this criterion is equivalent to cutting off the momentum of each fermion leg. In the LOFF case, it is not equivalent and is more appropriate. The choice we have made is not the only q-independent cutoff one might try. For example, we have also obtained results upon cutting off momenta outside a large ellipsoid in $\mathbf{p}$-space, confocal with the centers of the two Fermi spheres in Figure 2, but have found that this makes little difference relative to the simpler choice of the large sphere $|\mathbf{p}|<\Lambda$.

\section{Results}

We solve the gap equation (4.9) numerically (and analytically in the limit $\Delta_{A} \ll$ $\left.\delta \mu, q, \Delta_{0}\right)$ and calculate the LOFF state free energy as a function of $\delta \mu$ and $q$, for given coupling $G$, average chemical potential $\bar{\mu}$, and cutoff $\Lambda$. We vary $q$ to minimize the LOFF free energy, and compare it with that for the standard BCS pairing (4.10) to see which is favored. In this way we can map out the phase diagram for the three phases of pairing between the two species of quark: BCS, LOFF, and unpaired.

Note that the solution to the gap equation, the LOFF gap parameter $\Delta_{A}$, is not a gap in the spectrum of excitations. The quasiparticle dispersion relations (4.7) vary with the direction of the momentum, yielding gaps that vary from zero (for 

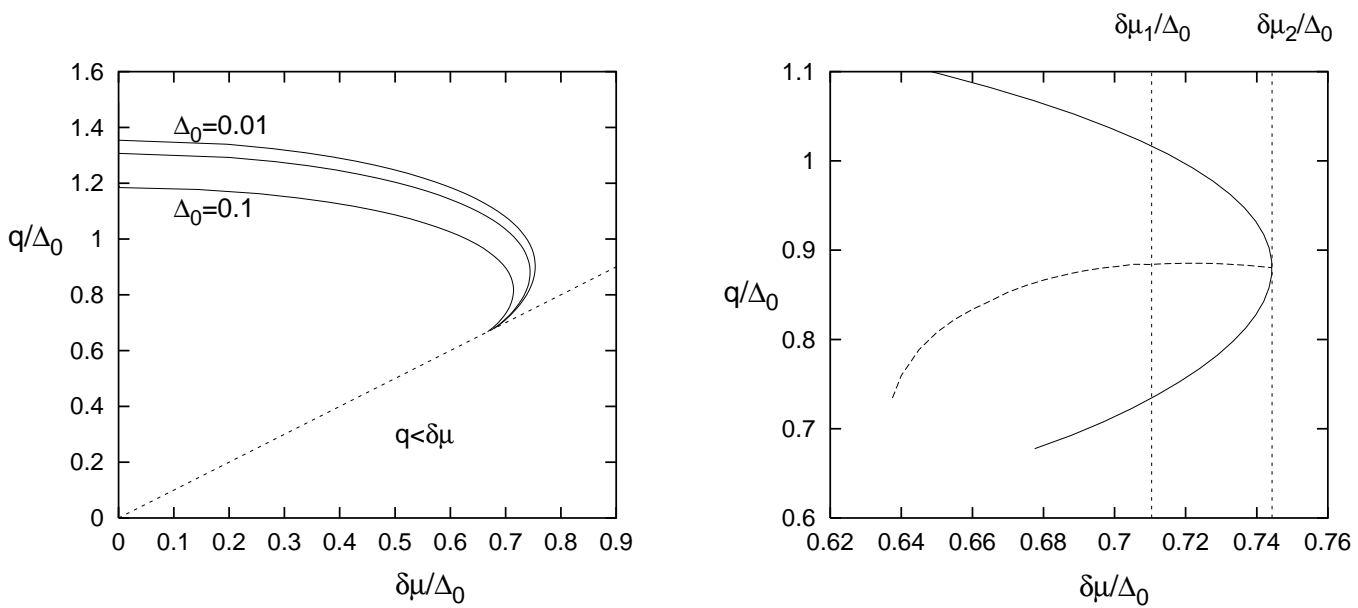

Figure 3: (a) The zero-gap curves for the LOFF state. To the right of a solid curve, there is no solution to the LOFF gap equation, to the left of the curve there is a solution, and on the curve the gap parameter is zero. The three curves are (from strongest to weakest coupling): $\Delta_{0}=0.1,0.04,0.01 \mathrm{GeV}$. The region $q<\delta \mu$ is complicated to describe [13], and solutions found in this region never give the lowest free energy state at a given $\delta \mu$. (b) Here, we choose $\Delta_{0}=0.04 \mathrm{GeV}$ and focus on the region near $\delta \mu_{2}$, the maximum value of $\delta \mu$ at which the LOFF state exists. The dashed curve shows the value of $|\mathbf{q}|$ which minimizes the free energy of the LOFF state at a given $\delta \mu$. $\delta \mu_{1}$, discussed below, is also indicated.

momenta on the edge of the blocking regions in phase space) up to a maximum of $\Delta_{A}$.

We will first discuss the range of $\delta \mu$ in which there exists a LOFF state as a local energy minimum. Later we will go on to study the competition between LOFF and $\mathrm{BCS}$, and see in what range of $\delta \mu$ the LOFF state is the global minimum. We expect the BCS state to be preferred when the mismatch $\delta \mu$ between the Fermi energies of the two species is small. When the mismatch is comparable to the BCS gap $\left(\delta \mu \sim \Delta_{0}\right)$ we expect a transition to LOFF, and at larger $\delta \mu$ we expect all pairing to cease. These expectations are largely borne out.

In general we fix $\Lambda=1 \mathrm{GeV}$ and $\bar{\mu}=0.4 \mathrm{GeV}$, and study different coupling strengths $G$ which we parameterize by the physical quantity $\Delta_{0}$, the BCS gap of Eq. (4.10) which increases monotonically with increasing $G$. When we wish to study the dependence on the cutoff, we vary $\Lambda$ while at the same time varying the coupling $G$ such that $\Delta_{0}$ is kept fixed. (This is in the same spirit as using a renormalization condition on a physical quantity - $\Delta_{0}$ - to fix the "bare" coupling$G$.) We expect that the relation between other physical quantities and $\Delta_{0}$ will be reasonably insensitive to variation of the cutoff $\Lambda$.

We wish to determine $\delta \mu_{2}$, the boundary separating the LOFF phase and the normal phase. The LOFF to unpaired phase transition is second order, so it occurs where the solution $\Delta_{A}$ to the LOFF gap equation (4.9) is zero. Setting $\Delta_{A}=0$ in the 
gap equation (4.10) yields an analytical expression relating $\delta \mu$ and $q$, for any given $G$ and $\Lambda$. In Figure 3 a we show the $\Delta_{A}=0$ curve for three couplings corresponding to $\Delta_{0}=0.1 \mathrm{GeV}$ (strong coupling), $\Delta_{0}=0.04 \mathrm{GeV}$ and $\Delta_{0}=0.01 \mathrm{GeV}$ (weak coupling). We have only drawn the zero-gap curve in the region where $q \geq \delta \mu$. We expect this to be the region of interest for LOFF pairing because when $q \geq \delta \mu$ the two spheres of Figure 2 do in fact intersect. We have verified that, as described in some detail in Ref. [13, there are regions of Figure a with $q<\delta \mu$ within which the LOFF gap equation (4.9) has (one or even two) nonzero solutions, but these solutions all correspond to phases whose free energy is either greater than that of the normal phase or greater than that of the BCS phase or both. Figure 3 shows that for a given coupling strength, parameterized by $\Delta_{0}$, there is a maximum $\delta \mu$ for which the LOFF state exists: we call it $\delta \mu_{2}$. For $\delta \mu>\delta \mu_{2}$, the mismatch of chemical potentials is too great for the LOFF phase to exist.

We see from Figure 3a that as the coupling gets weaker, $\delta \mu_{2} / \Delta_{0}$ gets gradually larger. (Of course, $\delta \mu_{2}$ itself gets smaller: the quantities plotted are $\delta \mu / \Delta_{0}$ and $\left.q / \Delta_{0}.\right)$ Note that in the $\Delta_{0} \rightarrow 0$ limit, the zero gap curve is essentially that shown in the figure for $\Delta_{0}=0.01 \mathrm{GeV}$, in agreement with the curve obtained at weak coupling by Fulde and Ferrell [13]. The fact that this curve ceases to move in the $\Delta_{0} \rightarrow 0$ limit means that $\delta \mu_{2} \rightarrow 0$ while $\delta \mu_{2} / \Delta_{0} \rightarrow$ const in this limit.

For $\delta \mu \rightarrow \delta \mu_{2}$ from below, we see from Figure 3 that there is a solution to the LOFF gap equation only at a single value of $q$. For example, at $\Delta_{0}=0.04 \mathrm{GeV}$ we find $q=0.880 \Delta_{0}=1.183 \delta \mu_{2}$ at $\delta \mu_{2}=0.744 \Delta_{0}$. (In agreement with Refs. [12, 13], in the weak coupling limit we find $q=0.906 \Delta_{0}=1.20 \delta \mu_{2}$ at $\delta \mu_{2}=0.754 \Delta_{0}$.) For any value of $\delta \mu<\delta \mu_{2}$, solutions to the LOFF gap equation exist for a range of $|\mathbf{q}|$. We must now find the value of $|\mathbf{q}|$ for which the free energy of the LOFF state is minimized. We obtain the free energy of the LOFF state at a point in Figure 3 by first solving the gap equation (4.9) numerically to obtain $\Delta_{A}$, and then using (4.4) and (4.6) to evaluate $\left\langle F_{0}+H_{I}\right\rangle$ given in (4.2) and (4.3). For each value of $\delta \mu<\delta \mu_{2}$ we can now determine which choice of $q$ yields the lowest free energy. The resulting "best- $q$ curve" curve is shown in Figure 3b for $\Delta_{0}=0.04 \mathrm{GeV}$.

Finally, for each point on the best- $q$ curve we ask whether the LOFF free energy at that $\delta \mu$ and (best) $q$ is more or less than the free energy of the BCS state at the same $\delta \mu$. In this way, we find $\delta \mu_{1}$ at which a first order phase transition between the LOFF and BCS states occurs. In Figure 1 we show the competition

\footnotetext{
${ }^{5}$ As a check on our determination of the best $q$, we have confirmed that the total momentum of the LOFF state with the best $q$ is zero, as must be the case for the ground state of the system at a given $\delta \mu$ (by a theorem attributed to Bloch [57). This is a powerful check, because it requires the net momentum of the unpaired quarks in the blocking regions (which is in the negative $z$ direction; see Figure 2) to be cancelled by the net momentum carried by the LOFF condensates. When, in future work, our ansatz is extended to describe a LOFF crystal rather than a single plane wave, this check will no longer be powerful. Once we go from $\Gamma \sim \exp (2 i \mathbf{q} \cdot \mathbf{r})$ to $\Gamma \sim \cos (2 \mathbf{q} \cdot \mathbf{r})$ or to a more involved crystalline pattern, the total momentum of the condensates and of the unpaired quarks will each be zero.
} 

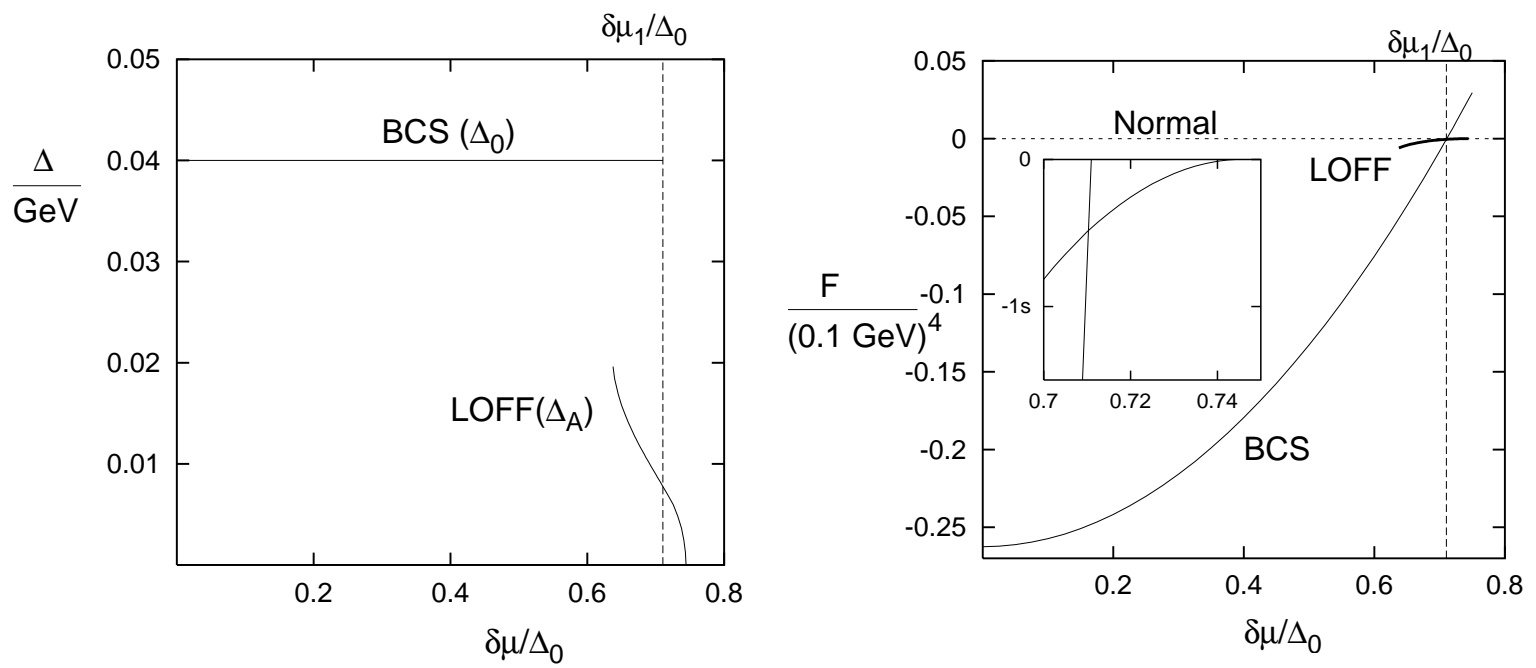

Figure 4: LOFF and BCS gaps and free energies as a function of $\delta \mu$, with coupling chosen so that $\Delta_{0}=40 \mathrm{MeV}$ and with $\bar{\mu}=0.4 \mathrm{GeV}, \Lambda=1 \mathrm{GeV}$. Free energies are measured relative to the normal state. At each $\delta \mu$ we have varied $q$ to find the best LOFF state. The vertical dashed line marks $\delta \mu=\delta \mu_{1}$, the value of $\delta \mu$ above which the LOFF state has lower free energy than BCS. The expanded inset (wherein $s=10^{-7} \mathrm{GeV}^{4}$ ) focuses on the region $\delta \mu_{1}<\delta \mu<\delta \mu_{2}$ where the LOFF state has the lowest free energy.

between the BCS and LOFF states as a function of the Fermi surface mismatch $\delta \mu$, for a fixed coupling corresponding to $\Delta_{0}=40 \mathrm{MeV}$. The LOFF state exists for $\delta \mu<\delta \mu_{2}=0.744 \Delta_{0}$. At each $\delta \mu<\delta \mu_{2}$, we plot the gap parameter and free energy characterizing the LOFF state with the best $q$ for that $\delta \mu$. Although the BCS gap $\Delta_{0}$ is larger than the LOFF gap $\Delta_{A}$, as $\delta \mu$ increases we see from Eq. (2.3) that the BCS state pays a steadily increasing free-energetic price for maintaining $p_{F}^{u}=p_{F}^{d}$, whereas the LOFF state pays no such price. We now see that the LOFF state has lower free energy than the BCS state for $\delta \mu>\delta \mu_{1}$, in this case $\delta \mu_{1}=0.7104 \Delta_{0}$. At $\delta \mu=\delta \mu_{1}$, the gap parameter is $\Delta_{A}=0.0078 \mathrm{GeV}=0.195 \Delta_{0}$. (Had we calculated $\delta \mu_{1}$ by comparing the BCS free energy with that of the unpaired state instead of with that of the LOFF state, we would have obtained $\delta \mu_{1}=0.711 \Delta_{0}$. As the inset to Figure 1 confirms, the BCS free energy varies so rapidly that this makes an almost imperceptible difference. In later figures, we therefore obtain $\delta \mu_{1}$ via the simpler route of comparing BCS vs. normal.) At the coupling corresponding to $\Delta_{0}=40$ $\mathrm{MeV}$, we have found that the LOFF state is favored over both the BCS state and the normal state in a "LOFF window" $0.710<\delta \mu / \Delta_{0}<0.744$.

With solutions to the gap equation in hand, we can obtain the $J=0$ condensate $\Gamma_{A}=G \Delta_{A}$ and the $J=1$ condensate $\Gamma_{B}$ given in Eq. (4.11). In Figure 5, we show both condensates within the LOFF window $\delta \mu_{1}<\delta \mu<\delta \mu_{2}$. We see first of all that $\Gamma_{B} \neq 0$, as advertised. For the choice of parameters in Figs. G and 5 we find $\Gamma_{B} / \Gamma_{A}$ 


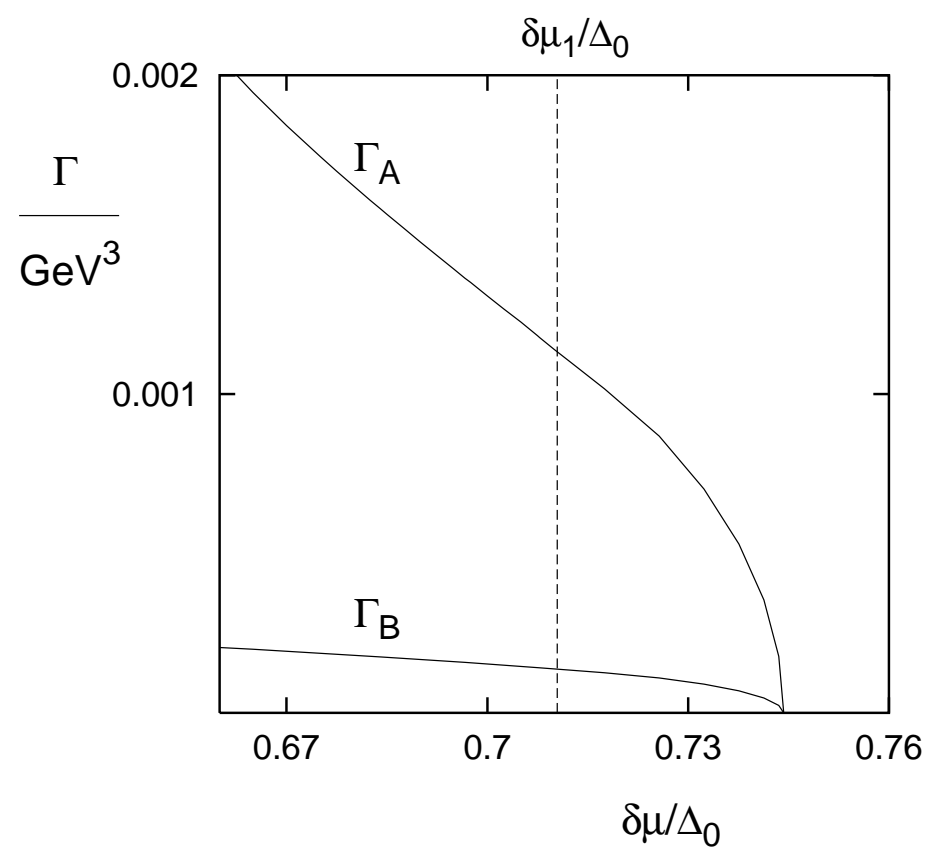

Figure 5: The two LOFF condensates $\Gamma_{A}(J=0)$ and $\Gamma_{B}(J=1)$ for the same choice of parameters as in Figure 1 . We focus on the region $\delta \mu_{1}<\delta \mu<\delta \mu_{2}$. For reference, in the BCS phase $\Gamma_{A}=\Delta_{0} / G=0.00583 \mathrm{GeV}^{3}$ and $\Gamma_{B}=0$.

essentially constant over the whole LOFF window, varying from 0.121 at $\delta \mu_{1}$ to 0.133 at $\delta \mu_{2}$. Increasing $\Delta_{0}$ tends to increase $\Gamma_{B} / \Gamma_{A}$, as does decreasing $\Lambda$. Second of all, we see that the phase transition at $\delta \mu=\delta \mu_{2}$, between the LOFF and normal phases, is second order in the mean-field approximation we employ throughout.

Near the second-order critical point $\delta \mu_{2}$, we can describe the phase transition with a Ginzburg-Landau effective potential. The order parameter for the LOFF-tonormal phase transition is

$$
\Phi(\mathbf{r})=-\frac{1}{2}\left\langle\epsilon_{i j} \epsilon_{\alpha \beta 3} \psi^{i \alpha}(\mathbf{r}) C \gamma_{5} \psi^{j \beta}(\mathbf{r})\right\rangle
$$

so that in the normal phase $\Phi(\mathbf{r})=0$, while in the LOFF phase $\Phi(\mathbf{r})=\Gamma_{A} e^{i 2 \mathbf{q} \cdot \mathbf{r}}$. Expressing the order parameter in terms of its Fourier modes $\tilde{\Phi}(\mathbf{k})$, we write the LOFF free energy (relative to the normal state) as

$$
F(\{\tilde{\Phi}(\mathbf{k})\})=\sum_{\mathbf{k}}\left(C_{2}\left(k^{2}\right)|\tilde{\Phi}(\mathbf{k})|^{2}+C_{4}\left(k^{2}\right)|\tilde{\Phi}(\mathbf{k})|^{4}+\mathcal{O}\left(|\tilde{\Phi}|^{6}\right)\right) .
$$

For $\delta \mu>\delta \mu_{2}$, all of the $C_{2}\left(k^{2}\right)$ are positive and the normal state is stable. Just below the critical point, all of the modes $\tilde{\Phi}(\mathbf{k})$ are stable except those on the sphere $|\mathbf{k}|=2 q_{2}$, where $q_{2}$ is the value of $|\mathbf{q}|$ at $\delta \mu_{2}$ (so that $q_{2} \simeq 1.2 \delta \mu_{2} \simeq 0.9 \Delta_{0}$ at weak coupling). In general, therefore, many modes on this sphere can become nonzero, giving a condensate with a complex crystal structure. We consider the simplest 
case of a plane wave condensate where only the one mode $\tilde{\Phi}\left(\mathbf{k}=2 \mathbf{q}_{2}\right)=\Gamma_{A}$ is nonvanishing. Dropping all other modes, we have

$$
F\left(\Gamma_{A}\right)=a\left(\delta \mu-\delta \mu_{2}\right)\left(\Gamma_{A}\right)^{2}+b\left(\Gamma_{A}\right)^{4}
$$

where $a$ and $b$ are positive constants. Finding the minimum-energy solution for $\delta \mu<\delta \mu_{2}$, we obtain simple power-law relations for the condensate and the free energy:

$$
\Gamma_{A}(\delta \mu)=K_{\Gamma}\left(\delta \mu_{2}-\delta \mu\right)^{1 / 2}, \quad F(\delta \mu)=-K_{F}\left(\delta \mu_{2}-\delta \mu\right)^{2} .
$$

These expressions agree well with the numerical results shown in Figs. 14and 5. The Ginzburg-Landau method does not specify the proportionality factors $K_{\Gamma}$ and $K_{F}$, but analytical expressions for these coefficients can be obtained in the weak coupling limit by explicitly solving the gap equation [51], yielding

$$
\begin{aligned}
G_{A} K_{\Gamma} & =2 \sqrt{\delta \mu_{2}} \sqrt{\left(q_{2} / \delta \mu_{2}\right)^{2}-1} \simeq 1.15 \sqrt{\Delta_{0}} \\
K_{F} & =\left(4 \bar{\mu}^{2} / \pi^{2}\right)\left(\left(q_{2} / \delta \mu_{2}\right)^{2}-1\right) \simeq 0.178 \bar{\mu}^{2} .
\end{aligned}
$$

Notice that because $\left(\delta \mu_{2}-\delta \mu_{1}\right) / \delta \mu_{2}$ is quite small, the power-law relations (5.4) are a good model of the system throughout the entire LOFF interval $\delta \mu_{1}<\delta \mu<\delta \mu_{2}$ where the LOFF phase is favored over the BCS phase. The Ginzburg-Landau expression (5.3) gives the free energy of the LOFF phase near $\delta \mu_{2}$, but it cannot be used to determine the location $\delta \mu_{1}$ of the first-order phase transition where the LOFF window terminates (locating the first-order point requires a comparison of LOFF and BCS free energies).

It is interesting to explore how the width of the LOFF window depends on the strength of the coupling, and to confirm that it is insensitive to the cutoff. We do this in Figure 6, where we plot $\delta \mu_{2} / \Delta_{0}$ (solid lines) and $\delta \mu_{1} / \Delta_{0}$ (dashed lines). The LOFF state is favored for $\delta \mu_{1} / \Delta_{0}<\delta \mu / \Delta_{0}<\delta \mu_{2} / \Delta_{0}$, i.e. between the solid and dashed curves in Figure 6. In the weak coupling limit, the LOFF window tends to $0.707<\delta \mu / \Delta_{0}<0.754$ and $\Delta_{A}$ at $\delta \mu_{1}$ tends to $0.23 \Delta_{0}$, as in Refs. [12, 13]. Note that if one takes the weak-coupling limit $\Delta_{0} \rightarrow 0$ at fixed $\delta \mu$, neither BCS nor LOFF pairing survives because $\delta \mu / \Delta_{0} \rightarrow \infty$. However, for any arbitrarily small but nonzero coupling, the LOFF phase is favored within a range of $\delta \mu$. Figure thus demonstrates that in an analysis of the LOFF state in the weak-coupling limit, it is convenient to keep $\delta \mu / \Delta_{0}$ fixed while taking $\Delta_{0} \rightarrow 0$. We see from Figure 6 that strong coupling helps the BCS state more than it helps the LOFF state. When the coupling gets strong enough, there is no longer any window of Fermi surface mismatch $\delta \mu$ in which the LOFF state occurs: the BCS state is always preferred.

The different lines of each type in Figure 6 are for different cutoffs and show that there is in fact little sensitivity to the cutoff. The $\Lambda$ dependence of $\delta \mu_{1} / \Delta_{0}$ and $\delta \mu_{2} / \Delta_{0}$ is mild for all values of $\Delta_{0}$ which are of interest, and is weakest for $\Delta_{0} \rightarrow 0$. This is because in that limit pairing can only occur very close to the unblocked ribbon of the ellipsoid of Fig. 2b, along which the integrand in the gap equation is 


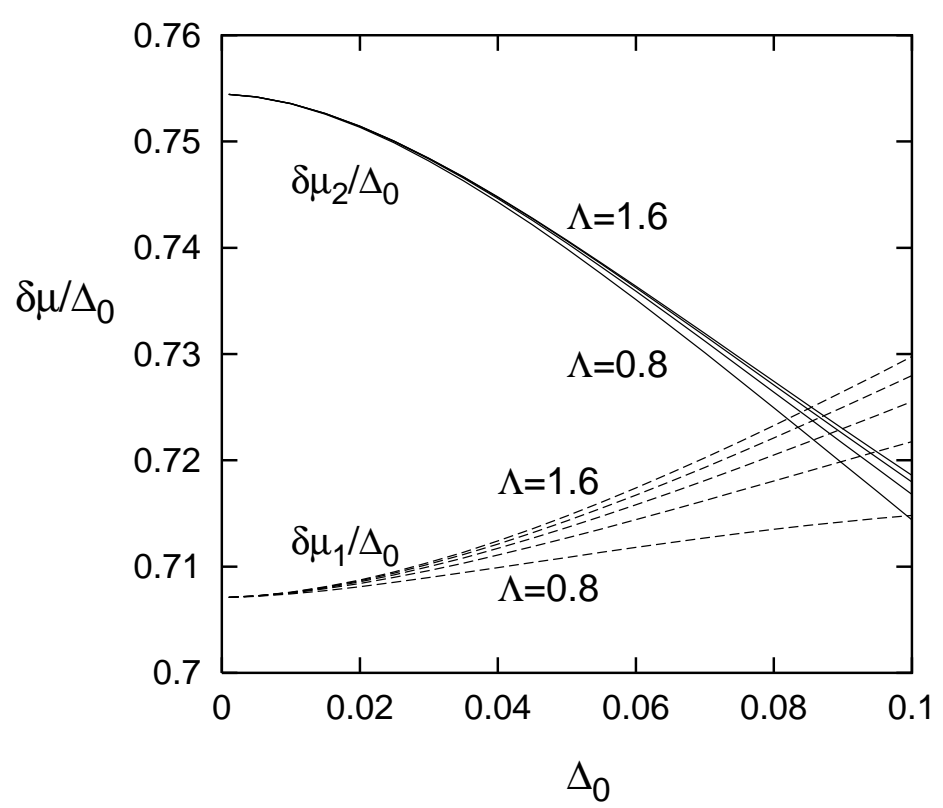

Figure 6: The interval of $\delta \mu$ within which the LOFF state occurs, as a function of the coupling (parameterized as usual by the BCS gap $\Delta_{0}$ ). Below the solid line, there is a LOFF state. Below the dashed line, the BCS state is favored. The different lines of each type correspond to different cutoffs $\Lambda=0.8 \mathrm{GeV}$ to $1.6 \mathrm{GeV} . \delta \mu_{1} / \Delta_{0}$ and $\delta \mu_{2} / \Delta_{0}$ show little cutoff-dependence, and the cutoff-dependence disappears completely as $\Delta_{0}, \delta \mu \rightarrow 0$.

singular and pairing is allowed. Thus most of the pairing region $\mathcal{P}$, and in particular the region near $\Lambda$, become irrelevant in this limit.

The one physical quantity which we have explored which does turn out to depend qualitatively on $\Lambda$ is the ratio $\Gamma_{B} / \Gamma_{A}$. Those quarks with momenta as large as $\Lambda$ which pair have momenta which are almost antiparallel, and so contribute much less to $\Gamma_{B}$ than to $\Gamma_{A}$. For this reason, the ratio $\Gamma_{B} / \Gamma_{A}$ is sensitive to the number of Cooper pairs formed at very large $\mathbf{p}$, and hence to the choice of $\Lambda$. As discussed above, pairing far from the favored ribbon in phase space becomes irrelevant for $\Delta_{0} \rightarrow 0$, and indeed in this limit we find that the $\Lambda$ dependence of $\Gamma_{B} / \Gamma_{A}$ decreases. However, for $\Delta_{0}=40 \mathrm{MeV}$ we find that changing $\Lambda$ from $1.2 \mathrm{GeV}$ to $0.8 \mathrm{GeV}$ increases $\Gamma_{B} / \Gamma_{A}$ by more than $50 \%$.

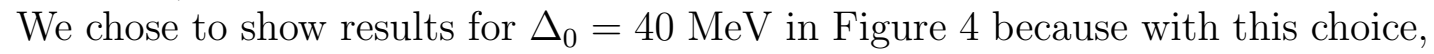
the LOFF window occurs at values of $\delta \mu$ comparable to that in the illustrative example (2.1): $\delta \mu=\frac{1}{2}\left(\mu_{d}-\mu_{u}\right)=27 \mathrm{MeV}$. Of course, neither $\delta \mu$ nor the value of $\Delta_{0}$ are accurately known for the quark matter which may exist within a compact star. Still, it seems possible that their ratio could be appropriate for the quark matter to be in the LOFF phase. If there is a range of radii within a compact star in which quark matter occurs with $\delta \mu_{1}<\delta \mu<\delta \mu_{2}$, this quark matter will be a crystalline color superconductor. 
In Figure 4 , the LOFF gap parameter $\Delta_{A}$ is $7.8 \mathrm{MeV}$ at $\delta \mu=\delta \mu_{1}$. It remains larger than typical neutron star temperatures $T_{\mathrm{ns}} \sim 1 \mathrm{keV}$ until very close to $\delta \mu=$ $\delta \mu_{2}$. Similarly, the LOFF free energy, which is $4.8 \times 10^{-8} \mathrm{GeV}^{4}=4.8 \times(10 \mathrm{MeV})^{4}$ at $\delta \mu=\delta \mu_{1}$, is much larger than $T_{\mathrm{ns}}^{4}$ throughout the LOFF window except very close to $\delta \mu=\delta \mu_{2}$. Furthermore, we shall see in Section 7.3 that the free energy of the LOFF state is of the right order to lead to interesting glitch phenomena.

\section{More general Hamiltonian and ansatz}

In Section 4 , we introduced the four-fermion interaction Hamiltonian $H_{I}$ of Eq. (4.1) with independent couplings $G_{E}$ and $G_{M}$ for the interactions which model the exchange of electric and magnetic gluons. It proves convenient to use the linear combinations

$$
\begin{aligned}
G_{A} & =\frac{1}{4}\left(G_{E}+3 G_{M}\right) \\
G_{B} & =\frac{1}{4}\left(G_{E}-G_{M}\right),
\end{aligned}
$$

of the coupling constants in terms of which the expectation value of $H_{I}$ in the LOFF state (3.2) becomes

$$
\left\langle H_{I}\right\rangle=-\frac{1}{2} G_{A} V\left(\left|\Gamma_{A}^{L}\right|^{2}+\left|\Gamma_{A}^{R}\right|^{2}\right)-\frac{1}{2} G_{B} V\left(\left|\Gamma_{B}^{L}\right|^{2}+\left|\Gamma_{B}^{R}\right|^{2}\right) .
$$

Thus, a positive coupling $G_{A}$ describes an attractive interaction which induces a $J=0$ condensate $\Gamma_{A}$. As we have seen, in the LOFF state this is necessarily accompanied by a $J=1$ condensate $\Gamma_{B}$. In our analysis to this point, we have set $G_{A}=G>0$ and $G_{B}=0$. We now discuss the general case, in which $G_{B} \neq 0$.

Before beginning, let us consider how to choose $G_{B} / G_{A}$ in order for our model Hamiltonian to be a reasonable toy model for QCD at nonzero baryon density. At zero density, of course, Lorentz invariance requires $G_{B}=0$. At high densities, on the other hand, electric gluons are screened while static magnetic gluons are not. (Magnetic gluons with nonzero frequency are damped.) We now know 18 that at asymptotically high densities it is in fact the exchange of magnetic gluons which dominates the pairing interaction. This suggests the choice $G_{E}=0$, corresponding to $G_{B} / G_{A}=-1 / 3$. At the accessible densities of interest to us, it is presumably not appropriate to neglect $G_{E}$ completely. Note also that the four-fermion interaction induced by instantons in QCD only yields interactions in flavor-antisymmetric channels. It results in an attractive interaction in the $J=0$ channel and no interaction in the $J=1$ channel. Thus, although the instanton interaction cannot be written in the form (4.1), for our purposes it can be thought of as adding a

contribution to $G_{A}$, but none to $G_{B}$. Hence our model is likely to best represent high density QCD for a ratio of couplings lying somewhere in the range

$$
-\frac{1}{3}<\frac{G_{B}}{G_{A}}<0
$$

We plot our results over a wider range of couplings below. 
Once $G_{B} \neq 0$ and there is an interaction in the $J=1$ channel, we expect, in addition to the $J=1$ condensate $\Gamma_{B}$, a $J=1$ gap parameter $\Delta_{B}$. The quasiparticle dispersion relations are then determined by $\Delta_{A}$ and $\Delta_{B}$, which are defined as

$$
\begin{aligned}
\Delta_{A} & =G_{A} \Gamma_{A} \\
\Delta_{B} & =G_{B} \Gamma_{B} .
\end{aligned}
$$

Following through the variational calculation as in Section 1 leads to the coupled gap equations:

$$
\begin{aligned}
\Delta_{A} & =\frac{2 G_{A}}{V} \sum_{\mathbf{p} \in \mathcal{P}} \frac{2 S_{A}\left(\Delta_{A} S_{A}+\Delta_{B} S_{B}\right)}{\sqrt{(|\mathbf{q}+\mathbf{p}|+|\mathbf{q}-\mathbf{p}|-2 \bar{\mu})^{2}+4\left(\Delta_{A} S_{A}+\Delta_{B} S_{B}\right)^{2}}} \\
\Delta_{B} & =\frac{2 G_{B}}{V} \sum_{\mathbf{p} \in \mathcal{P}} \frac{2 S_{B}\left(\Delta_{A} S_{A}+\Delta_{B} S_{B}\right)}{\sqrt{(|\mathbf{q}+\mathbf{p}|+|\mathbf{q}-\mathbf{p}|-2 \bar{\mu})^{2}+4\left(\Delta_{A} S_{A}+\Delta_{B} S_{B}\right)^{2}}} \\
S_{A} & =\sin \left(\frac{1}{2} \beta_{A}(\mathbf{p})\right) \\
S_{B} & =\sin \left(\frac{1}{2} \beta_{B}(\mathbf{p})\right)
\end{aligned}
$$

with $\beta_{A}(\mathbf{p})=\alpha_{u}(\mathbf{p})+\alpha_{d}(\mathbf{p}), \beta_{B}(\mathbf{p})=\alpha_{u}(\mathbf{p})-\alpha_{d}(\mathbf{p})$ defined in terms of the angles in Figure 11. The pairing region $\mathcal{P}$ is still defined by (4.8) but with new quasiparticle dispersion relations obtained from Eqs. (4.7) with $\Delta_{A}^{2} S_{A}^{2}$ replaced by $\left(\Delta_{A} S_{A}+\Delta_{B} S_{B}\right)^{2}$.

For $G_{B}=0$, the coupled equations (6.5) reduce to Eqs. (4.9) and (4.11). Note that if, instead, $G_{B}>0$ and $G_{A}=0$, we find an attractive interaction in the $J=1$ channel in Eq. (6.1) and no interaction in the $J=0$ channel. Analysis of Eqs. (6.5) in this case yields a nonzero value of $\Delta_{B}$, while $\Delta_{A}=0$ even though $\Gamma_{A} \neq 0$. The geometry of the LOFF pairs requires $\Gamma_{A} \neq 0$ when $\Gamma_{B} \neq 0$.

Rather than describing how every Figure in Section 5 changes when $G_{B} \neq 0$, we choose to focus on the question of how the interval of $\delta \mu$ within which the LOFF state occurs (the LOFF window) changes as a function of $G_{B} / G_{A}$. To further simplify the presentation, we specialize to the weak-coupling limit in which $\Delta_{0} \rightarrow 0$. This means that, as in Figure 6, the LOFF window is independent of the cutoff $\Lambda$.

We show the dependence of the LOFF window on $G_{B} / G_{A}$ in Figure 7. The lower boundary $\delta \mu=\delta \mu_{1}$ is, as in Section 5, the same (up to a very small correction) as the $\delta \mu$ at which the BCS and normal states have equal free energies. We find the upper boundary $\delta \mu=\delta \mu_{2}$ by first dividing Eqs. (6.5) by $\Delta_{A}$ and then looking for a value of $\delta \mu$ at which $\Delta_{A} \rightarrow 0$ and $\Delta_{B} \rightarrow 0$ but $\Delta_{A} / \Delta_{B}$ remains nonzero. As before, this defines a zero-gap curve, and $\delta \mu_{2}$ is the maximum value of $\delta \mu$ reached by this curve.

We find that the lower boundary $\delta \mu_{1}$ is completely unaffected by the value of $G_{B}$, since the BCS state is purely $J=0$. So in the weak-coupling limit we obtain the result of Section 5, $\delta \mu_{1} / \Delta_{0}=0.707$, independent of $G_{B} / G_{A}$. In contrast, $\delta \mu_{2}$, the upper boundary of the LOFF window, increases with increasing $G_{B}$. This is understandable: the LOFF state always produces a $J=1$ condensate, so we expect 


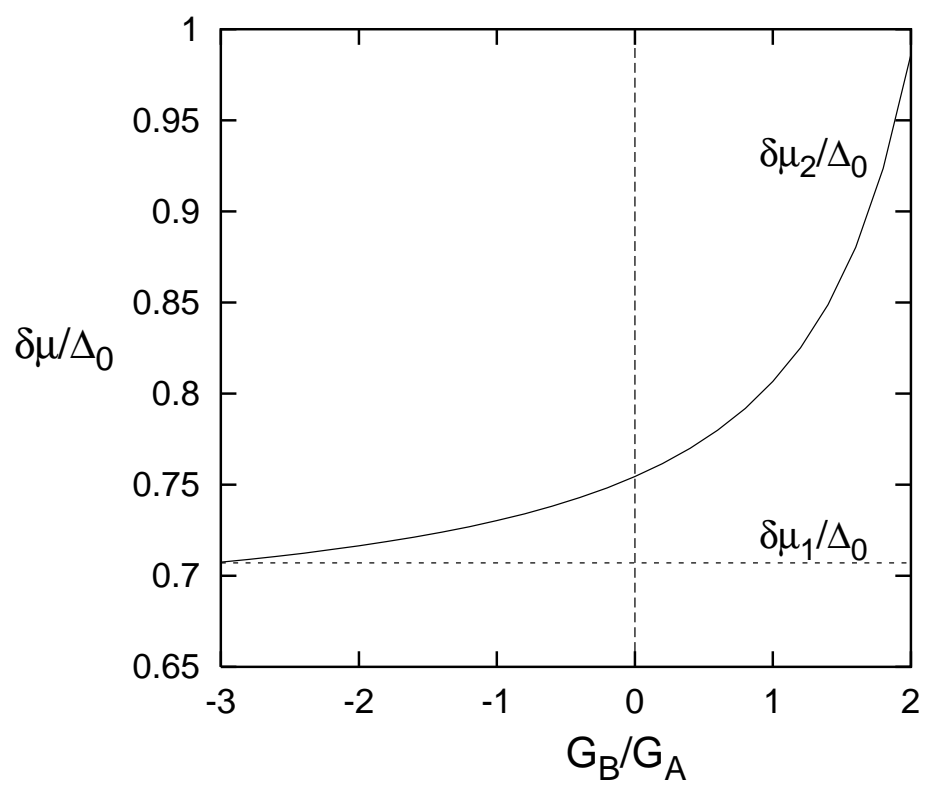

Figure 7: The interval of $\delta \mu$ in which the LOFF state is favored at weak coupling, as a function of the ratio of couplings $G_{B} / G_{A}$. Below the solid line, there is a LOFF state. Below the dashed line, the ordinary BCS state is favored. $G_{B}=0$ corresponds to the Lorentz-invariant interaction with $G_{E}=G_{M}$. QCD at high density is likely best described by a coupling in the range $-\frac{1}{3}<G_{B} / G_{A}<0$.

it to be fortified by $G_{B}>0$ and penalized by $G_{B}<0$. There is no analogue of this behavior in an electron superconductor [12, 13], where there can be no $J=1$ condensate. Our $J=1$ condensate affects the gap equation and free energy only if $G_{B} \neq 0$; for this reason, our weak coupling results are in agreement with those of LOFF [12, 13] only if $G_{B}=0$, as in Section 5. The effect of a coupling $G_{B}$ in the physically interesting range (6.3) is to reduce the LOFF window, but only slightly.

In both this Section and the previous one, we have calculated $\delta \mu_{2}$ by examining the competition between LOFF pairing and no pairing. Should we instead have considered the competition between LOFF pairing and the formation of $\langle u u\rangle$ and $\langle d d\rangle$ condensates, each at their respective Fermi surface, each with $\mathbf{q}=0$ ? Assuming as usual that the color antisymmetric channel is the most attractive one, flavor symmetric pairing requires spin-symmetric pairing, i.e. $J=1$ [58]. Within the model and ansatz that we have considered, the question is easily answered. If we choose $G_{A}>0$ and $G_{B}=0$, as in Sections 3 to 5, there is no interaction in the spin-symmetric, flavor-symmetric, color-antisymmetric channel. If we strengthen magnetic gluon exchange relative to electric gluon exchange by choosing $G_{B}<0$, the interaction in this channel is repulsive. We have confirmed this by evaluating the expectation value of $H_{I}$ in a state with spatially uniform $J=1$ pairing and $\left\langle u C \sigma^{0 i} u\right\rangle$ condensate (obtained by using two $u$ creation operators in the ansatz (3.2), setting $\delta \mu=0$ as appropriate for $\langle u u\rangle$ pairing, setting $\mathbf{q}=0$ and removing the blocking 
regions). We find that $G_{A}$ gives no interaction in this channel and $G_{B}<0$ is repulsive. Thus, for the same reason that the LOFF window shrinks for $G_{B}<0$, there can be no $\langle u u\rangle$ or $\langle d d\rangle$ pairing. However, the scenario is apparently different at asymptotically high density: it has been shown by Schäfer [58 that long-range single-gluon exchange does in fact induce pairing in this $J=1$ channel. (The longrange interaction emphasizes near-collinear scattering which is attractive for both electric and magnetic gluons.) For either a pointlike interaction with $G_{B}>0$ or a long-range interaction dominated by near-collinear scattering, we therefore expect competition between LOFF pairing and $\langle u u\rangle$ and $\langle d d\rangle$ pairing, since the latter would then be favored for $\delta \mu>\delta \mu_{2}$.

Our ansatz only contains $L L$ and $R R$ pairing. We leave a complete analysis of the generalization to $L R$ pairing to future work. We have, however, constructed the ansatz for spatially uniform $L R$ pairing with $\delta \mu=0$ and $\mathbf{q}=0$. We find that the interaction in this $J=1$ channel is attractive if $G_{E}+G_{M}>0$ and is independent of the linear combination of couplings $G_{E}-3 G_{M}$. The $J=1$ channel with LR

pairing yields a $\left\langle u C \gamma^{i} u\right\rangle$ condensate instead of the $J=1$ condensate $\left\langle u C \sigma^{0 i} u\right\rangle$ obtained for the case of $L L$ and $R R$ pairing. In agreement with Ref. [58] we find that magnetic gluon exchange, with $G_{E}=0$ and $G_{M}>0$, is attractive in the $\left\langle u C \gamma^{i} u\right\rangle$ channel. Note that in the nonrelativistic limit $\left\langle u C \gamma^{i} u\right\rangle$ and $\left\langle u C \sigma^{0 i} u\right\rangle$ are equivalent $J=1$ condensates. In the relativistic setting relevant in quark matter, we find that pointlike interactions in these two channels have opposite sign.

We have set up the gap equation describing a spatially uniform $\left\langle u C \gamma^{i} u\right\rangle$ condensate and solved it for $G_{E}=G_{M}=G, \Delta_{0}=40 \mathrm{MeV}, \mu_{u}=0.4 \mathrm{GeV}$, and $\Lambda=1 \mathrm{GeV}$. We find a gap of $8 \mathrm{keV}$ and a free energy which is about five orders of magnitude smaller than that of the LOFF phase. (If we choose $G_{E}=0$ and $G_{M}>0$, the interaction is still attractive but the gap is even smaller.) Therefore, even though for $\delta \mu>\delta \mu_{2}$ we expect LR pairing and consequent $\left\langle u C \gamma^{i} u\right\rangle$ and $\left\langle d C \gamma^{i} d\right\rangle$ condensates, the resulting condensation energy is so small that it is a good approximation to neglect these condensates in the evaluation of $\delta \mu_{2}$, as we have done. We leave for future work a complete analysis of the competition between the LOFF phase (with an ansatz extended to allow $L R$-LOFF pairing) and the spatially uniform $\left\langle u C \gamma^{i} u\right\rangle$ condensate.

\section{Conclusions, future work and astrophysical implications}

\subsection{Conclusions}

We have studied the formation of a rotational-symmetry-breaking LOFF state involving pairing between two flavors of quark whose chemical potentials differ by $2 \delta \mu$. This state is characterized by a gap parameter and a diquark condensate, but not by an energy gap in the dispersion relation. In the LOFF state, each Cooper pair carries momentum $2 \mathbf{q}$ with $|\mathbf{q}| \approx 1.2 \delta \mu$. The condensate and gap parameter vary in space with wavelength $\pi /|\mathbf{q}|$. 
We focused primarily on an NJL-type four-fermion interaction with the quantum numbers of single gluon exchange. In the limit of weak coupling (BCS gap $\Delta_{0} \ll \mu$ ) the LOFF state is favored for values of $\delta \mu$ which satisfy $\delta \mu_{1}<\delta \mu<\delta \mu_{2}$, where $\delta \mu_{1} / \Delta_{0}=0.707$ and $\delta \mu_{2} / \Delta_{0}=0.754$. The LOFF gap parameter decreases from $0.23 \Delta_{0}$ at $\delta \mu=\delta \mu_{1}$ to zero at $\delta \mu=\delta \mu_{2}$. These are the same results found by LOFF in their original analysis. Except for very close to $\delta \mu_{2}$, the critical temperature above which the LOFF state melts will be much higher than typical neutron star temperatures. At stronger coupling the LOFF gap parameter decreases relative to $\Delta_{0}$ and the window of $\delta \mu / \Delta_{0}$ within which the LOFF state is favored shrinks. The window grows if the interaction is changed to weight electric gluon exchange more heavily than magnetic gluon exchange.

Because it violates rotational invariance by involving Cooper pairs whose momenta are not antiparallel, the quark matter LOFF state necessarily features nonzero condensates in both the $J=0$ and $J=1$ channels. Both condensates are present even if there is no interaction in the $J=1$ channel. In this case, however, the $J=1$ condensate does not affect the quasiparticle dispersion relations; that is, the $J=1$ gap parameter vanishes. If there is an attraction in the $J=1$ channel (as, for example, if the strength of the electric gluon interaction is increased) the size of the LOFF window increases.

The quark matter which may be present within a compact star will be in the crystalline color superconductor (LOFF) state if $\delta \mu / \Delta_{0}$ is in the requisite range. For $\delta \mu$ as in the illustrative example (2.1), this occurs if the gap $\Delta_{0}$ which characterizes the uniform color superconductor present at smaller values of $\delta \mu$ is about $40 \mathrm{MeV}$. This is in the middle of the range of present estimates. Both $\delta \mu$ and $\Delta_{0}$ vary as a function of density and hence as a function of radius in a compact star. Although it is too early to make quantitative predictions, the numbers are such that crystalline color superconducting quark matter may very well occur in a range of radii within a compact star. It is therefore worthwhile to consider the consequences.

\subsection{Future work}

The prospect of spontaneous violation of translational and rotational symmetry in dense quark matter is very exciting. In the final pages of this paper we will begin to explore one particularly interesting consequence: glitch behavior in quark matter within compact stars. First, however, we list a number of direct extensions of our work, several of which are prerequisites to a quantitative exploration of the astrophysical consequences of crystalline color superconducting quark matter in compact stars.

1. We have restricted ourselves to two flavors of quark, and varied $\delta \mu$ freely. It is crucial to look at more realistic examples, imposing charge neutrality and weak equilibrium, and including the strange quark. We expect a LOFF phase wherever $\langle u s\rangle,\langle d s\rangle$ or $\langle u d\rangle$ pairs approach their unpairing transitions, but 
this must be verified quantitatively. Further generalizations would include bare quark masses and spontaneous generation of constituent quark masses by chiral condensation.

2. It would be valuable to complement our NJL-model study with a controlled calculation using one gluon exchange in the asymptotically high density limit. There are two reasons why this is worthwhile. First, it will allow a controlled analysis without model assumptions, albeit one of quantitative value only at extremely high densities. In particular, this would allow a better estimation of the relative magnitude of the $J=1$ and $J=0$ condensates, which was the one feature which we found to depend strongly on the choice of cutoff in our model. Second, quark-quark scattering by the exchange of a gluon at weak coupling is dominated by small-angle scattering, whereas in an NJL model of the type we have used this is not the case. This can actually affect the sign of the interaction in the $J=1$ channel and perhaps thereby increase the range of the LOFF window, as we pointed out in the previous section. Moreover, it is known that the LOFF window is much wider in one dimension than in three [49, and since the three-dimensional physics at asymptotically high densities can be treated as a sum of one-dimensional theories [21, 26], we have another reason to suspect that the LOFF window may be wider at asymptotically high densities than our present analysis would suggest.

3. As we have discussed at length in Section 6, it would be of interest to extend our treatment to include pairing between quarks of the same flavor and pairing between quarks of opposite chirality.

4. Perhaps the most crucial unresolved issue is the question of what crystal structure the LOFF phase chooses. Larkin and Ovchinnikov concluded that the condensate varies in space like $\cos (2 \mathbf{q} \cdot \mathbf{r})$, forming a one-dimensional standing wave with nodal planes spaced every $\pi /(2|\mathbf{q}|)$. The competition between this planar structure and one with, say, a cubic or body-centered-cubic crystal structure is subtle. In two dimensions, the answer depends sensitively on the temperature [41]; in three dimensions, it is apparently still unresolved even in the original LOFF context [42]. In the QCD context, with the added complication of a $J=1$ condensate, it will be quite interesting to determine what pattern is favored.

5. Finally, it would be very interesting to investigate the astrophysical consequences of the LOFF phase. Since it occurs in a range of $\delta \mu$, one would expect that quark matter stars could contain a layer of crystalline LOFF condensate. In the next subsection, we take some preliminary steps in this investigation. 


\subsection{Looking ahead to astrophysical consequences}

Many pulsars have been observed to glitch. Glitches are sudden jumps in rotation frequency $\Omega$ which may be as large as $\Delta \Omega / \Omega \sim 10^{-6}$, but may also be several orders of magnitude smaller. The frequency of observed glitches is statistically consistent with the hypothesis that all radio pulsars experience glitches [59]. Glitches are thought to originate from interactions between the rigid crust, somewhat more than a kilometer thick in a typical neutron star, and rotational vortices in the neutron superfluid. The inner kilometer of the crust consists of a rigid lattice of nuclei immersed in a neutron superfluid [60]. Because the pulsar is spinning, the neutron superfluid (both within the inner crust and deeper inside the star) is threaded with a regular array of rotational vortices. As the pulsar's spin gradually slows due to emission of electromagnetic radiation, these vortices must gradually move outwards since the rotation frequency of a superfluid is proportional to the density of vortices. Deep within the star, the vortices are free to move outwards. In the crust, however, the vortices are pinned by their interaction with the nuclear lattice. What happens next varies from model to model. Perhaps the vortices exert sufficient force on the crust to tear it apart, resulting in a sudden breaking and rearrangement of the crust and a change in the moment of inertia 61. Perhaps a large cluster of vortices within the inner crust builds up enough outward pressure to overcome the pinning force, suddenly becomes unpinned, and moves macroscopically outward [62, 63, 64, 65, 66, 67, 68]. This sudden decrease in the angular momentum of the superfluid within the crust results in a sudden increase in angular momentum of the rigid crust itself, and hence a glitch. Perhaps, due to interactions between neutron vortices and proton flux tubes, the neutron vortices pile up just inside the inner crust before suddenly coming unpinned [69]. Although the models differ in important respects, all agree that the fundamental requirements are the presence of rotational vortices in a superfluid and the presence of a rigid structure which impedes the motion of vortices and which encompasses enough of the volume of the pulsar to contribute significantly to the total moment of inertia. [

Although it is premature to draw quantitative conclusions, it is interesting to speculate that some glitches may originate deep within a pulsar which features a quark matter core, in a region of that core in which the color superconducting quark matter is in a LOFF crystalline color superconductor phase. The first prerequisite for a quantitative answer to whether this may occur is to repeat our analysis in the more general context of three-flavor quark matter with a nonzero strange quark mass $M_{s}$, to estimate over what range of densities LOFF phases may arise, as either $\langle u d\rangle,\langle u s\rangle$ or $\langle d s\rangle$ condensates approach their unpairing transitions. Comparison to existing models which describe how $p_{F}^{u}, p_{F}^{d}$ and $p_{F}^{s}$ vary within a quark matter

\footnotetext{
${ }^{6}$ The first model of glitches which was proposed [70] relies on the cracking and settling of the neutron star crust ("starquakes") as the neutron star spins down. This model does not require the presence of rotational vortices. However, this model fails to explain the magnitude and frequency of glitches in the Vela pulsar [67, 68].
} 
core in a neutron star [38] would then permit an estimate of how much the LOFF region contributes to the moment of inertia of the pulsar. Furthermore, a three flavor analysis is required to determine whether the LOFF phase is a superfluid. If the only pairing is between $u$ and $d$ quarks, this 2SC phase is not a superfluid [3, 9], whereas if all three quarks pair in some way, a superfluid is obtained [6, 9. 9

Henceforth, we suppose that the LOFF phase is a superfluid, which means that if it occurs within a pulsar it will be threaded by an array of rotational vortices. It is reasonable to expect that these vortices will be pinned in a LOFF crystal, in which the diquark condensate varies periodically in space. Indeed, one of the suggestions for how to look for a LOFF phase in terrestrial electron superconductors relies on the fact that the pinning of magnetic flux tubes (which, like the rotational vortices of interest to us, have normal cores) is expected to be much stronger in a LOFF phase than in a uniform BCS superconductor [71].

A real calculation of the pinning force experienced by a vortex in a crystalline color superconductor must await the determination of the crystal structure of the LOFF phase. We can, however, attempt an order of magnitude estimate along the same lines as that done by Anderson and Itoh [62] for neutron vortices in the inner crust of a neutron star. In that context, this estimate has since been made quantitative [72, 66, 68]. With parameters chosen as in Figure 4 , we find that at $\delta \mu=\delta \mu_{1}$ the LOFF phase is favored over the normal state by a free energy $F_{\mathrm{LOFF}} \sim 5 \times(10 \mathrm{MeV})^{4}$ and the spacing between nodes in the LOFF crystal is $b=\pi /(2|\mathbf{q}|) \sim 9 \mathrm{fm}$. The thickness of a rotational vortex is given by the correlation length $\xi \sim 1 / \Delta_{A} \sim 25 \mathrm{fm}$. All these numbers are quite uncertain, but we will use them for the present. In the context of crustal neutron superfluid vortices, there are three distinct length scales: the vortex thickness $\xi$, the lattice spacing between nuclei $b$, and $R$, the radius of the individual nuclei. (The condensate vanishes within regions of size $R$ separated by spacing $b$.) In the LOFF phase, the latter two length scales are comparable: since the condensate varies like $\cos (\pi r / b)$ it is as if $R \sim b$. The fact that these length scales are similar in the LOFF phase will complicate a quantitative calculation of the pinning energy; it makes our order of magnitude estimation easier, however. The pinning energy is the difference between the energy of a section of vortex of length $b$ which is centered on a node of the LOFF crystal vs. one which is centered on a maximum of the LOFF crystal. It is of order

$$
E_{p} \sim F_{\mathrm{LOFF}} b^{3} \sim 4 \mathrm{MeV} .
$$

The resulting pinning force per unit length of vortex is of order

$$
f_{p} \sim \frac{E_{p}}{b^{2}} \sim \frac{4 \mathrm{MeV}}{80 \mathrm{fm}^{2}} .
$$

A complete calculation will be challenging because $b<\xi$, and is likely to yield an $f_{p}$ which is somewhat less than that we have obtained by dimensional analysis 66, 68.

\footnotetext{
${ }^{7}$ As an aside, note that the crystalline chiral condensate [56] (due to particle-hole pairing which may form at sufficiently strong coupling or at very large $N_{c}$ ) is not a superfluid.
} 
Note that our estimate of $f_{p}$ is quite uncertain both because it is only based on dimensional analysis and because the values of $\Delta_{A}, b$ and $F_{\text {LOFF }}$ are uncertain. (We know the values of all the ratios $\Delta_{A} / \Delta_{0}, \delta \mu / \Delta_{0}, q / \Delta_{0}$ and consequently $b \Delta_{0}$ quite accurately in the LOFF phase. It is of course the value of the BCS gap $\Delta_{0}$ which is uncertain.) It is therefore premature to compare our crude result to the results of serious calculations of the pinning of crustal neutron vortices as in Refs. [72, 66, 68]. It is nevertheless remarkable that they prove to be similar: the pinning energy of neutron vortices in the inner crust is [66]

$$
E_{p} \approx 1-3 \mathrm{MeV}
$$

and the pinning force per unit length is 66, 67

$$
f_{p} \sim \frac{E_{p}}{b \xi} \approx \frac{1-3 \mathrm{MeV}}{(25-50 \mathrm{fm})(4-20 \mathrm{fm})},
$$

where the form of this expression is appropriate because $\xi<b$. Perhaps, therefore, glitches occurring in a region of crystalline color superconducting quark matter may yield similar phenomenology to those occurring in the inner crust.

The reader may be concerned that a glitch deep within the quark matter core of a neutron star may not be observable: the vortices within the crystalline color superconductor region suddenly unpin and leap outward; this loss of angular momentum is compensated by a gain in angular momentum of the layer outside the LOFF region; how quickly, then, does this increase in angular momentum manifest itself at the surface of the star as a glitch? If the LOFF layer is the outer layer of the quark matter core - not unreasonable since the chemical potential differences will be larger here than deeper inside the quark matter - there is no problem. The LOFF glitch speeds up the nucleon superfluid outside the quark matter core, and the rotation of this superfluid is coupled to the rotation of the outer crust on very short time scales 73 . This rapid coupling, due to electron scattering off vortices and the fact that the electron fluid penetrates throughout the star, is usually invoked to explain that the core nucleon superfluid speeds up quickly after a crustal glitch: the only long relaxation time is that of the vortices within the inner crust [73]. Here, we invoke it to explain that the outer crust speeds up rapidly after a LOFF glitch has accelerated the quark matter at the base of the nucleon superfluid. After a glitch in the LOFF region, the only long relaxation times are those of the vortices in the $\mathrm{LOFF}$ region and in the inner crust.

A quantitative theory of glitches originating within quark matter in a LOFF phase must await the further microscopic calculations sketched in Section 7.2. In particular, an understanding of points 1 and 4 of Section 7.2 is a mandatory prerequisite. However, our rough estimate of the pinning force on rotational vortices in a LOFF region suggests that this force may be comparable in magnitude to that on vortices in the inner crust of a conventional neutron star, which yields glitches in accord with those observed in pulsars. This is surely strong motivation for further investigation. 
Perhaps the most interesting consequence of these speculations arises in the context of compact stars made entirely of strange quark matter. The work of Witten [74] and Farhi and Jaffe [75] raised the possibility that strange quark matter may be energetically stable relative to nuclear matter even at zero pressure. If this is the case it raises the question whether observed compact stars-pulsars, for example - are strange quark stars [76, 77] rather than neutron stars. A conventional neutron star may feature a core made of strange quark matter, as we have been discussing above. Strange quark stars, on the other hand, are made (almost) entirely of quark matter with either no hadronic matter content at all or with a thin crust, of order one hundred meters thick, which contains no neutron superfluid [77, 78]. The nuclei in this thin crust are supported above the quark matter by electrostatic forces; these forces cannot support a neutron fluid. Because of the absence of superfluid neutrons, and because of the thinness of the crust, no successful models of glitches in the crust of a strange quark star have been proposed. Since pulsars are observed to glitch, the apparent lack of a glitch mechanism for strange quark stars has been the strongest argument that pulsars cannot be strange quark stars [79, 80, 81]. This conclusion must now be revisited.

Madsen's conclusion [34] that a strange quark star is prone to r-mode instability due to the absence of damping must also be revisited, since the relevant fluid oscillations may be damped within or at the boundary of a region of crystalline color superconductor.

The quark matter in a strange quark star, should one exist, would be a color superconductor. Depending on the mass of the star, the quark number densities increase by a factor of about two to ten in going from the surface to the center [77]. This means that the chemical potential differences among the three quarks will vary also, and there could be a range of radii within which the quark matter is in a crystalline color superconductor phase. This raises the possibility of glitches in strange quark stars. Because the variation in density with radius is gradual, if a shell of LOFF quark matter exists it need not be particularly thin. And, we have seen, the pinning forces may be comparable in magnitude to those in the inner crust of a conventional neutron star. It has recently been suggested (for reasons unrelated to our considerations) that certain accreting compact stars may be strange quark stars [82], although the evidence is far from unambiguous [83]. In contrast, it has been thought that, because they glitch, conventional radio pulsars cannot be strange quark stars. Our work questions this assertion by raising the possibility that glitches may originate within a layer of quark matter which is in a crystalline color superconducting state.

\section{Acknowledgements}

We are grateful to C. Nayak for pointing out Refs. [12 and [13 to us at the Aspen Center for Physics more than one year ago. We are grateful to him and to 
P. Bedaque, J. Berges, I. Bombaci, D. Blaschke, D. Chakrabarty, R. Jaffe, J. Madsen, D. Psaltis, S.-J. Rey, M. Ruderman, T. Schäfer, A. Sedrakian, E. Shuster, D. Son, M. Stephanov, I. Wasserman, F. Weber and F. Wilczek for helpful discussions. We are grateful to the Department of Energy's Institute for Nuclear Theory at the University of Washington for its hospitality and support during the completion of much of this work. This work is supported in part by the U.S. Department of Energy (D.O.E.) under cooperative research agreement \#DF-FC02-94ER40818. The work of $\mathrm{KR}$ is supported in part by a DOE OJI Award and by the Alfred P. Sloan Foundation. The work of JB is supported by a DOD National Defense Science and Engineering Graduate Fellowship.

\section{References}

[1] B. Barrois, Nucl. Phys. B129 (1977) 390. S. Frautschi, Proceedings of workshop on hadronic matter at extreme density, Erice 1978. B. Barrois, "Nonperturbative effects in dense quark matter", Cal Tech PhD thesis, UMI 79-04847-mc (1979).

[2] D. Bailin and A. Love, Phys. Rept. 107 (1984) 325, and references therein.

[3] M. Alford, K. Rajagopal and F. Wilczek, Phys. Lett. B422, 247 (1998) hepph/9711395.

[4] R. Rapp, T. Schäfer, E. V. Shuryak and M. Velkovsky, Phys. Rev. Lett. 81, 53 (1998) hep-ph/9711396.

[5] J. Berges and K. Rajagopal, Nucl. Phys. B538, 215 (1999) hep-ph/9804233.

[6] M. Alford, K. Rajagopal and F. Wilczek, Nucl. Phys. B537, 443 (1999) hepph/9804403.

[7] For recent reviews, see K. Rajagopal, to appear in Proceedings of Quark Matter '99, hep-ph/9908360; F. Wilczek, to appear in Proceedings of PANIC '99, hepph/9908480; T. Schäfer, nucl-th/9911017; M. Alford, to appear in Proceedings of TMU-Yale, Dec 1999 hep-ph/0003185.

[8] A. M. Clogston, Phys. Rev. Lett. 9, 266 (1962); B. S. Chandrasekhar, App. Phys. Lett. 1, 7 (1962).

[9] M. Alford, J. Berges and K. Rajagopal, Nucl. Phys. B558, 219 (1999) hepph/9903502.

[10] T. Schäfer and F. Wilczek, Phys. Rev. D60, 074014 (1999) hep-ph/9903503.

[11] P. Bedaque, hep-ph/9910247. 
[12] A. I. Larkin and Yu. N. Ovchinnikov, Zh. Eksp. Teor. Fiz. 47, 1136 (1964); translation: Sov. Phys. JETP 20, 762 (1965).

[13] P. Fulde and R. A. Ferrell, Phys. Rev. 135, A550 (1964).

[14] G. W. Carter and D. Diakonov, Phys. Rev. D60, 016004 (1999) hepph/9812445.

[15] R. Rapp, T. Schäfer, E. V. Shuryak and M. Velkovsky, Annals Phys. 280, 35 (2000) hep-ph/9904353.

[16] N. Evans, S. D. Hsu and M. Schwetz, Nucl. Phys. B551, 275 (1999) hepph/9808444; Phys. Lett. B449, 281 (1999) hep-ph/9810514.

[17] T. Schäfer and F. Wilczek, Phys. Lett. B450, 325 (1999) [hep-ph/9810509].

[18] D. T. Son, Phys. Rev. D59, 094019 (1999) hep-ph/9812287.

[19] T. Schäfer and F. Wilczek, Phys. Rev. D60, 114033 (1999) hep-ph/9906512.

[20] R. D. Pisarski and D. H. Rischke, Phys. Rev. Lett. 83, 37 (1999) nucl th/9811104; Phys. Rev. D60, 094013 (1999) nucl-th/9903023; Phys. Rev. D61, 051501 (2000) nucl-th/9907041; R. D. Pisarski and D. H. Rischke, Phys. Rev. D61, 074017 (2000) nucl-th/9910056.

[21] D. K. Hong, Phys. Lett. B473, 118 (2000) hep-ph/9812510; hep-ph/9905523.

[22] D. K. Hong, V. A. Miransky, I. A. Shovkovy and L. C. Wijewardhana, Phys. Rev. D61, 056001 (2000) hep-ph/9906478.

[23] W. E. Brown, J. T. Liu and H. Ren, Phys. Rev. D61, 114012 (2000) hepph/9908248; Phys. Rev. D62, 054016 (2000) hep-ph/9912409; Phys. Rev. D62, 054013 (2000) [hep-ph/0003199.

[24] S. D. Hsu and M. Schwetz, Nucl. Phys. B572, 211 (2000) hep-ph/9908310.

[25] T. Schäfer, Nucl. Phys. B575, 269 (2000) [hep-ph/9909574.

[26] S. Beane, P. Bedaque and M. Savage, nucl-th/0004013; S. Beane and P. Bedaque, nucl-th/0005052.

[27] I. A. Shovkovy and L. C. Wijewardhana, Phys. Lett. B470, 189 (1999) hepph/9910225.

[28] N. Evans, J. Hormuzdiar, S. D. Hsu and M. Schwetz, Nucl. Phys. B581, 391 (2000) hep-ph/9910313.

[29] K. Rajagopal and E. Shuster, to appear in Phys. Rev. D, hep-ph/0004074. 
[30] M. Alford, J. Berges and K. Rajagopal, Nucl. Phys. B571, 269 (2000) thep$\mathrm{ph} / 9910254$.

[31] D. Blaschke, D.M. Sedrakian and K.M. Shahabasyan, astro-ph/9904395; astroph/9911349.

[32] D. Page, M. Prakash, J. Lattimer and A. Steiner, hep-ph/0005094.

[33] D. Blaschke, T. Klaehn and D.N. Voskresensky, astro-ph/9908334.

[34] J. Madsen, Phys. Rev. Lett. 85, 10 (2000).

[35] L. Bildsten and G. Ushomirsky, astro-ph/9911155.

[36] G. Carter and S. Reddy, hep-ph/0005228.

[37] T. Schäfer and F. Wilczek, Phys. Rev. Lett. 82, 3956 (1999) hep-ph/9811473].

[38] N. K. Glendenning, Phys. Rev. D46, 1274 (1992); N. K. Glendenning, Compact Stars (Springer-Verlag, 1997); F. Weber, J. Phys. G. Nucl. Part. Phys. 25, R195 (1999).

[39] G. Sarma, Phys. Chem. Solids 24, 1029 (1963).

[40] A. Sedrakian and U. Lombardo, Phys. Rev. Lett. 84, 602 (2000).

[41] H. Shimahara, J. Phys. Soc. Jpn. 67, 736 (1998) cond-mat/9711017.

[42] A. I. Buzdin and H. Kachkachi, Phys. Lett. A225, 341 (1997).

[43] L. W. Gruenberg and L. Gunther, Phys. Rev. Lett. 16, 996 (1966).

[44] L. G. Aslamazov, Sov. Phys. JETP 28, 773 (1969).

[45] S. Takada, Prog. Theor. Phys. 43, 27 (1970).

[46] K. Gloos et al., Phys. Rev. Lett. 70, 501 (1993).

[47] G. Yin and K. Maki, Phys. Rev. B 48, 650 (1993); M. R. Norman, Phys. Rev. Lett. 71, 3391 (1993); H. Schimanski et al. Physica B 199, 125 (1994).

[48] L. N. Bulaevskii, Zh. Eksp. Teor. Fiz. 65, 1278 (1973), translation: Sov. Phys. JETP 38, 634 (1974); H. Shimahara, Phys. Rev. B 50, 12760 (1994); H. Burkhardt and D. Rainer, Ann. Physik 3, 181 (1994); G. Murthy and R. Shankar, J. Phys. Cond. Matt. 7, 9155 (1995); H. Shimahara and D. Raine, J. Phys. Soc. Jpn. 66, 3591 (1997); K. Yang and S. L. Sondhi, Phys. Rev. B 57, 8566 (1998); U. Klein, D. Rainer and H. Shimahara, J. Low. Temp. Phys. 118, 91 (2000) cond-mat/9909124; D. G. Agterberg and K. Yang, condmat/0006344. 
[49] A. I. Buzdin and V. V. Tugushev Zh. Eksp. Teor. Fiz. 85, 735 (1983), translation: Sov. Phys. JETP 58, 428 (1983); A. I. Buzdin and S. V. Polonskii, Zh. Eksp. Teor. Fiz. 93, 747 (1987), translation: Sov. Phys. JETP 66, 422 (1987); N. Dupuis, Phys. Rev. B 51, 9074 (1995).

[50] M. S. Nam et al., J. Phys. Cond. Matt. 11, L477 (1999); S. Manalo and U. Klein, J. Phys. Cond. Matt. 28, L471 (2000) cond-mat/0006327.

[51] S. Takada and T. Izuyama, Prog. Theor. Phys. 41, 635 (1969).

[52] D. T. Son and M. A. Stephanov, hep-ph/0005225.

[53] D. V. Deryagin, D. Y. Grigoriev and V. A. Rubakov, Int. J. Mod. Phys. A7, 659 (1992).

[54] E. Shuster and D. T. Son, Nucl. Phys. B573, 434 (2000) hep-ph/9905448.

[55] B. Park, M. Rho, A. Wirzba and I. Zahed, Phys. Rev. D62, 034015 (2000) hep-ph/9910347.

[56] R. Rapp, E. Shuryak and I. Zahed, hep-ph/0008207.

[57] F. London, Superfluids, Vol. I, Dover, New York, 1960, pg. 143.

[58] T. Schäfer, hep-ph/0006034.

[59] M. A. Alpar and C. Ho, Mon. Not. R. Astron. Soc. 204, 655 (1983). For a recent review, see A.G. Lyne in Pulsars: Problems and Progress, S. Johnston, M. A. Walker and M. Bailes, eds., 73 (ASP, 1996).

[60] J. Negele and D. Vautherin, Nucl. Phys. A207, 298 (1973).

[61] M. Ruderman, Astrophys. J. 382, 587 (1991); M. Ruderman, T. Zhu, and K. Chen, Astrophys. J. 492, 267 (1998); and references therein.

[62] P. W. Anderson and N. Itoh, Nature 256, 25 (1975).

[63] P. W. Anderson et al, Phil. Mag. A 45, 227 (1982).

[64] M. A. Alpar, P. W. Anderson, D. Pines and J. Shaham, Astrophys. J. 249, L29 (1981).

[65] M. A. Alpar, P. W. Anderson, D. Pines and J. Shaham, Astrophys. J. 276, 325 (1984).

[66] M. A. Alpar, P. W. Anderson, D. Pines and J. Shaham, Astrophys. J. 278, 791 (1984). 
[67] For reviews, see D. Pines and A. Alpar, Nature 316, 27 (1985); D. Pines, in Neutron Stars: Theory and Observation, J. Ventura and D. Pines, eds., 57 (Kluwer, 1991); M. A. Alpar, in The Lives of Neutron Stars, M. A. Alpar et al., eds., 185 (Kluwer, 1995).

[68] For more recent developments and references to further work, see R. I. Epstein and G. Baym, Astrophys. J. 387, 276 (1992); M. A. Alpar, H. F. Chau, K. S. Cheng and D. Pines, Astrophys. J. 409, 345 (1993); B. Link and R. I. Epstein, Astrophys. J. 457, 844 (1996).

[69] A. Sedrakian and J. M. Cordes, Mon. Not. R. Astron. Soc. 307, 365 (1999), and references therein.

[70] M. A. Ruderman, Nature 223, 597 (1969); G. Baym and D. Pines, Ann. Phys. 66, 816 (1971).

[71] R. Modler et al., Phys. Rev. Lett. 76, 1292 (1996).

[72] M. A. Alpar, Astrophys. J. 213, 527 (1977).

[73] M. A. Alpar, S. A. Langer and J. A. Sauls, Astrophys. J. 282, 533 (1984).

[74] E. Witten, Phys. Rev. D 30, 272 (1984).

[75] E. Farhi and R. L. Jaffe, Phys. Rev. D 30, 2379 (1984).

[76] P. Haensel, J. L. Zdunik and R. Schaeffer, Astron. Astrophys. 160, 121 (1986).

[77] C. Alcock, E. Farhi and A. Olinto, Phys. Rev. Lett. 57, 2088 (1986); Astrophys. J. 310, 261 (1986).

[78] N. K. Glendenning and F. Weber, Astrophys. J. 400, 647 (1992).

[79] A. Alpar, Phys. Rev. Lett. 58, 2152 (1987).

[80] J. Madsen, Phys. Rev. Lett. 61, 2909 (1988).

[81] R. R. Caldwell and J. L. Friedman, Phys. Lett. B264, 143 (1991).

[82] X.-D. Li, I. Bombaci, M. Dey, J. Dey, E. P. J. van den Heuvel, Phys. Rev. Lett. 83, 3776 (1999); X.-D. Li, S. Ray, J. Dey, M. Dey, I. Bombaci, Astrophys. J. 527, L51 (1999); B. Datta, A. V. Thampan, I. Bombaci, astro-ph/9912173; I. Bombaci, astro-ph/0002524.

[83] D. Psaltis and D. Chakrabarty, Astrophys. J. 521, 332 (1999); D. Chakrabarty, Phys. World 13, No. 2, 26 (2000). 